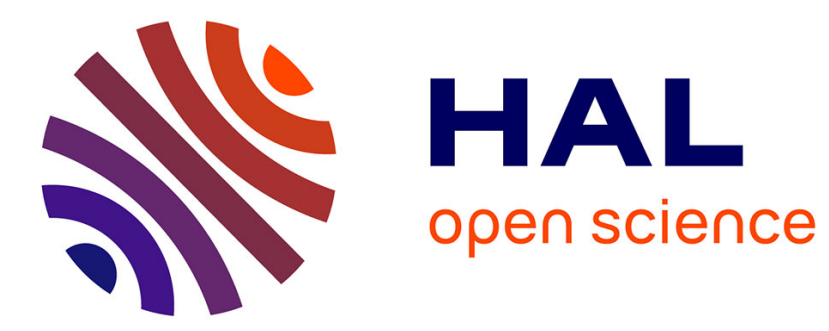

\title{
Dynamic analysis and suppressing chaotic impacts of a two-degree-of-freedom oscillator with a clearance
}

\author{
Guanwei Luo, Li Ma, Xiaohong Lv
}

\section{To cite this version:}

Guanwei Luo, Li Ma, Xiaohong Lv. Dynamic analysis and suppressing chaotic impacts of a twodegree-of-freedom oscillator with a clearance. Nonlinear Analysis: Real World Applications, 2009, 10 (2), pp.756-778. 10.1016/j.nonrwa.2007.11.002 . hal-01634313

\section{HAL Id: hal-01634313 https://hal.science/hal-01634313}

Submitted on 14 Nov 2017

HAL is a multi-disciplinary open access archive for the deposit and dissemination of scientific research documents, whether they are published or not. The documents may come from teaching and research institutions in France or abroad, or from public or private research centers.
L'archive ouverte pluridisciplinaire HAL, est destinée au dépôt et à la diffusion de documents scientifiques de niveau recherche, publiés ou non, émanant des établissements d'enseignement et de recherche français ou étrangers, des laboratoires publics ou privés. 


\title{
Dynamic analysis and suppressing chaotic impacts of a two-degree-of-freedom oscillator with a clearance
}

\author{
Guanwei Luo ${ }^{\mathrm{a}, *}, \mathrm{Li} \mathrm{Ma}^{\mathrm{b}}$, Xiaohong $\mathrm{Lv}^{\mathrm{a}}$ \\ a School of Mechatronic Engineering, Lanzhou Jiaotong University, Lanzhou 730070, PR China \\ ${ }^{\mathrm{b}}$ School of Mathematics, Physics and Software Engineering, Lanzhou Jiaotong University, Lanzhou, 730070, PR China
}

\begin{abstract}
A two-degree-of-freedom impact oscillator is considered. The maximum displacement of one of the masses is limited to a threshold value by the symmetrical rigid stops. Impacts between the mass and the stops are described by an instantaneous coefficient of restitution. Dynamics of the system is studied with special attention to periodic-impact motions and bifurcations. Period-one double-impact symmetrical motion and transcendental impact Poincaré map of the system is derived analytically. Stability and local bifurcations of the period-one double-impact symmetrical motions are analyzed by using the impact Poincaré map. The Lyapunov exponents in the vibratory system with impacts are calculated by using the transcendental impact map. The influence of the clearance and excitation frequency on symmetrical double-impact periodic motion and bifurcations is analyzed. A series of other periodic-impact motions are found and the corresponding bifurcations are analyzed. For smaller values of clearance, periodone double-impact symmetrical motion usually undergoes pitchfork bifurcation with decrease in the forcing frequency. For large values of the clearance, period-one double-impact symmetrical motion undergoes Neimark-Sacker bifurcation with decrease in the forcing frequency. The chattering-impact vibration and the sticking phenomena are found to occur in the region of low forcing frequency, which enlarges the adverse effects such as high noise levels, wear and tear and so on. These imply that the dynamic behavior of this system is very rich and complex, varying from different types of periodic motions to chaos, even chatteringimpacting vibration and sticking. Chaotic-impact motions are suppressed to minimize the adverse effects by using external driving force, delay feedback and feedback-based method of period pulse.
\end{abstract}

Keywords: Vibration; Impact; Periodic motion; Bifurcation; Suppressing chaos

\section{Introduction}

Vibrating systems with clearances between the moving parts are frequently encountered in technical applications. Impacts occur when the amplitudes of vibration of some parts of the system exceed critical values. Some examples are linkage machines, heat exchangers, fuel rods in nuclear power plants, rolling railway wheelset, piping systems, gear transmissions and so on. Impacts inevitably occur for the vibrating systems with clearances or gaps, but they

\footnotetext{
* Corresponding author. Fax: +86931 4938613.

E-mail addresses: luogw@mail.lzjtu.cn, luogw@ hotmail.com (G.W. Luo).
} 
are undesirable as they bring about failures, strain, shorter service life and increased noise levels. It is important to be able to accurately model the dynamics of an impacting system, so as to enlarge the profitable effects such as optimum design and high reliability, to minimize adverse effects such as high noise levels, pitting, wear and tear and so on. Compared with single impact, the nonlinear dynamics of vibro-impact systems is more complicated. The trajectories of such systems in phase space have discontinuities caused by the impacts. Consequently, the presence of the nonlinearity and discontinuity complicates the dynamic analysis of such systems considerably, but they can be described theoretically and numerically by discontinuities in good agreement with reality. The broad interest in analyzing and understanding the performance of such systems is reflected by a still increasing amount of investigations devoted to this area. Several methods of the theoretical analysis have been developed and different models of impacts have been assumed in the past several years. Stability and bifurcations of different types of impact oscillators were reported in Refs. [1-11]. Blazejczyk-Okolewska et al. [12] provided much information of the fundamental nature that broadens the scope of knowledge on the motion of mechanical systems with impacts. A special feature of impacting systems is the instability caused by grazing bifurcation. The first important work in this area was done by Nordmark [13]. This work has been further expanded by thorough investigations of two-dimensional maps, where some universal behavior has been found [14-23]. Based on the local singularity theory, Luo and Gegg [24] provided a comprehensive investigation of grazing motions in the dry-friction oscillator for a better understanding of the grazing mechanism of such a discontinuous system. Pavlovskaia and Wiercigroch [25-28] developed a series of mathematical models of impact systems with drift and revealed very complex dynamical behavior of such systems. Souza and Caldas [29] applied a model-based algorithm for the calculation of the spectrum of the Lyapunov exponents of attractors of mechanical systems with impacts. Peterka [30] found chaotic motion of an intermittency type of the impact oscillator appearing near segments of saddle-node stability boundaries of subharmonic motions with two different impacts in motion period. Luo [31] presented an idealized, piecewise linear system to model the vibration of gear transmission systems, and predicted analytically the periodic motions in a generalized, piecewise linear oscillator with perfectly plastic impacts. Wagg [32,33] analyzed the chattering impact and rising phenomena which occur in the stick solutions of impact oscillators. Luo and Gegg [34] developed the force criteria for stick and non-stick motions in harmonically forced, friction-induced oscillators from the local theory of non-smooth dynamical systems on connectable domains. Several effective methods of controlling chaos and experimental analysis have been developed in Refs. [35-42]. Hu [36] presented how to control the chaos of dynamical systems with discontinuous vector field through the paradigm of a harmonically forced oscillator having a set-up elastic stop. Lee and Yan [37] studied the algorithms of position control of impact oscillator and demonstrated synchronization of two impact oscillators. Souza et al. stabilized desired unstable periodic orbits, embedded in the chaotic invariant sets of mechanical systems with impacts, by applying a small and precise perturbation on an available control parameter [38], and investigated the influence of structural nonlinearity of a simple cantilever beam impacting system on its dynamic responses close to grazing incidence by means of numerical simulation [39]. Zinjade and Mallik [40] designed and fabricated an experimental setup of a single-degree-of-freedom friction-driven oscillator with an attached impact damper, and investigated the control of friction-driven oscillations by using an impact damper. The effects of mass ratio, coefficient of restitution and clearance on the performance of the impact damper have been investigated analytically and the results are verified by numerical integration. Zhao and Wang [41] proposed several feedback control methods to suppress chaotic behavior of an impact damper. Experimental study of base excited symmetrically piecewise linear oscillator was performed by Wiercigroch and Sin [42]. Along with the basic research into vibro-impact dynamics, a wide range of impacting models have been applied to simulate and analyze engineering systems operating within bounded dynamic responses. For example, in wheel-rail impacts of railway coaches [43,44], vibrating hammer [45], pile driver [46], Jeffcott rotor with bearing clearance [47-49], excited pendula with impacts [50], impact dampers [51-55], gears [31,56,57], etc., impacting models have proved to be useful [58].

A two-degree-of-freedom impact oscillator with a clearance is considered in the paper. The maximum displacement of one of the masses is limited to a threshold value by the symmetrical rigid stops. Dynamics of the system is studied with special attention to periodic-impact motions and bifurcations. Period-one double-impact symmetrical motion and transcendental impact map of the system are derived analytically. Stability and local bifurcations of the period-one double-impact symmetrical motions are analyzed by using the impact Poincaré map. The Lyapunov exponents in the impact oscillator with a clearance are calculated by using the transcendental impact map. The influence of the clearance and excitation frequency on the dynamics of the system is analyzed. The bifurcation regularity of periodone double-impact symmetrical motion is revealed. The chattering-impact vibration and the sticking phenomena are 


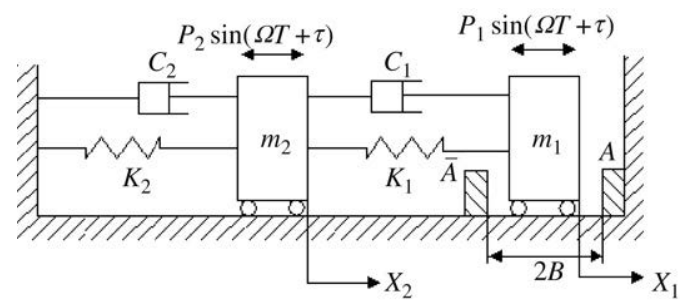

Fig. 1. Schematic of a harmonically excited system having symmetrical rigid stops.

found to occur in the region of low forcing frequency. These imply that the dynamics of this system is very complex, varying from different types of periodic motions to chaos, even chattering-impacting vibration and sticking. Chaoticimpact motions are suppressed to minimize the adverse effects by using external driving force, delay feedback and feedback-based method of period pulse.

\section{Mechanical model of a harmonically excited system having symmetrical rigid stops}

A two-degree-of-freedom system having symmetrically placed rigid stops and subjected to periodic excitation is shown schematically in Fig. 1. Displacements of the masses $M_{1}$ and $M_{2}$ are represented by $X_{1}$ and $X_{2}$, respectively. The masses are connected to the supporting base by linear springs with stiffness $K_{1}$ and $K_{2}$, and linear viscous dashpots with damping constants $C_{1}$ and $C_{2}$. Damping in the mechanical model is assumed as proportional damping. The excitations on both masses are harmonic with amplitudes $P_{1}$ and $P_{2}$. The excitation frequency $\Omega$ and the phase $\tau$ are the same for both masses. The masses move only in the horizontal direction. For small forcing amplitudes the system will undergo simple oscillations and behave as a linear system. As the amplitude is increased, the mass $M_{1}$ eventually begins to hit the stops and the motion becomes nonlinear (the other mass is not allowed to impact any stop). The impact is described by a coefficient of restitution $r$.

The motion processes of the system, between consecutive impacts occurring at the stop $A$, are considered. Between any two consecutive impacts, the time $T$ is always set to zero directly at the starting point (the stop $A$ ), and the phase angle $\tau$ is used only to make a suitable choice for the origin of time in the calculation. The state of the vibroimpact system, immediately after impact, has become new initial conditions in the subsequent process of the motion. Consequently, the motion of this system is a combination of smooth motions governed by linear differential equation interrupted by a series of non-smooth impacts. The dynamic analysis can be considered in a non-dimensional form, and all parameters and variables are assumed to be non-dimensional, which are introduced by

$$
\begin{gathered}
\mu_{m}=\frac{M_{2}}{M_{1}+M_{2}}, \quad \mu_{k}=\frac{K_{2}}{K_{1}+K_{2}}, \quad \mu_{c}=\frac{C_{2}}{C_{1}+C_{2}}, \quad f_{20}=\frac{P_{2}}{P_{1}+P_{2}}, \quad \omega=\Omega \sqrt{\frac{M_{1}}{K_{1}}}, \\
\zeta=\frac{C_{1}}{2 \sqrt{K_{1} M_{1}}}, \quad \delta=\frac{B K_{1}}{P_{1}+P_{2}}, \quad t=T \sqrt{\frac{K_{1}}{M_{1}}}, \quad x_{i}=\frac{X_{i} K_{1}}{P_{1}+P_{2}} .
\end{gathered}
$$

The governing equations of the system away from the impact can be expressed as

$$
\begin{aligned}
& {\left[\begin{array}{cc}
1 & 0 \\
0 & \frac{\mu_{m}}{1-\mu_{m}}
\end{array}\right]\left\{\begin{array}{l}
\ddot{x}_{1} \\
\ddot{x}_{2}
\end{array}\right\}+\left[\begin{array}{cc}
2 \zeta & -2 \zeta \\
-2 \zeta & \frac{2 \zeta}{1+\mu_{c}}
\end{array}\right]\left\{\begin{array}{l}
\dot{x}_{1} \\
\dot{x}_{2}
\end{array}\right\}+\left[\begin{array}{cc}
1 & -1 \\
-1 & \frac{1}{1-\mu_{k}}
\end{array}\right]\left\{\begin{array}{l}
x_{1} \\
x_{2}
\end{array}\right\}} \\
& =\left\{\begin{array}{c}
1-f_{20} \\
f_{20}
\end{array}\right\} \sin (\omega t+\tau), \quad\left|x_{1}\right|<\delta .
\end{aligned}
$$

An impact occurs wherever $\left|x_{1}(t)\right|=\delta$. This type of impact may be modelled using an instantaneous coefficient of restitution rule such that

$$
\dot{x}_{1 A+}=-r \dot{x}_{1 A-}, \quad\left(x_{1}=\delta\right), \quad \dot{x}_{1 \bar{A}+}=-r \dot{x}_{1 \bar{A}-}, \quad\left(x_{1}=-\delta\right) .
$$

In Eqs. (2) and (3), a dot (.) denotes differentiation with respect to the non-dimensional time $t$, the subscript minus sign denotes the states just before the impact and the subscript plus sign denotes the states just after the impact. 


\section{Impact Poincaré map and period-one double-impact symmetrical motion}

The impacting system is conveniently studied by the use of an impact Poincaré map derived from the equations of motion, supplemented by transition conditions at the instants of impacts. Here each iterate of this map corresponds to the mass $M_{1}$ impacting the stop $A$ once. The state of the vibro-impact system, just immediately after the impact occurring at the stop $A$, is chosen as the Poincaré section, i.e., $\sigma=\left\{\left(x_{1}, \dot{x}_{1}, x_{2}, \dot{x}_{2}, \theta\right) \in \boldsymbol{R}^{4} \times \boldsymbol{S}, x_{1}=\delta, \dot{x}_{1}=\dot{x}_{1+}\right\}$. As a result, the impact Poincaré map is expressed briefly by

$$
X^{\prime}=f(v, X),
$$

where $X \in \boldsymbol{R}^{4}, v$ is a bifurcation parameter, $v \in \boldsymbol{R}^{1}, X=\left(\dot{x}_{1+}, x_{2}, \dot{x}_{2}, \tau\right)^{\mathrm{T}}, X^{\prime}=\left(\dot{x}_{1+}^{\prime}, x_{2}^{\prime}, \dot{x}_{2}^{\prime}, \tau^{\prime}\right)^{\mathrm{T}}$.

The periodic motions of the vibro-impact system are characterized by the symbol $N-p-q$, where $q$ and $p$ are the numbers of impacts occurring respectively at the stops $A$ and $\bar{A}$, and $N$ is the number of the forcing cycles. In this section, the periodic motion of the model, with two symmetrical impacts per force cycle, is emphasized, which is called the period-one double-impact symmetrical motion, i.e., 1-1-1 symmetrical motion. The special periodic motion means that if the dimensionless time $t$ is set to zero directly just after an impact occurring at the stop $A$, it becomes $2 \pi / \omega$ just before the next impact occurring at the same stop. It certainly takes the same time for the mass $M_{1}$ to move from the stop $A$ to $\bar{A}$ and from the stop $\bar{A}$ to $A$, i.e., $t_{1}=t_{2}=\pi / \omega$. After the origin of $t$-coordinate is displaced to an impact point, the determination of period-one double-impact symmetrical motion is based on the fact that it satisfies the following set of periodicity and matching conditions

$$
\left[\begin{array}{c}
x(0) \\
\dot{x}(0)
\end{array}\right]=-\left[\begin{array}{c}
x(\pi / \omega)_{+} \\
\dot{x}(\pi / \omega)_{+}
\end{array}\right]=D\left[\begin{array}{c}
x(2 \pi / \omega) \\
\dot{x}(2 \pi / \omega)
\end{array}\right]=\left[\begin{array}{c}
x_{0} \\
\dot{x}_{0}
\end{array}\right], \quad\left[\begin{array}{l}
x(\pi / \omega)_{-} \\
\dot{x}(\pi / \omega)_{-}
\end{array}\right]=-\left[\begin{array}{c}
x(2 \pi / \omega) \\
\dot{x}(2 \pi / \omega)
\end{array}\right],
$$

where $D=\operatorname{diag}\left[d_{i}\right],\left(d_{i}=1, i=1,2,4 ; d_{3}=-r\right), x(t)=\left(x_{1}(t), x_{2}(t)\right)^{\mathrm{T}}, x_{0}=\left(\delta, x_{20}\right)^{\mathrm{T}}, \dot{x}_{0}=\left(\dot{x}_{1+}, \dot{x}_{20}\right)^{\mathrm{T}}$.

Combining the general solution of Eq. (2) with the set of periodicity and matching conditions, we can find the totalanalytical expressions for period-one double-impact symmetrical motion of the system. Inserting the time $t=0$ into the periodic solution, we can obtain the fixed point $X^{*}=\left(\dot{x}_{1+}, x_{10}, \dot{x}_{2+}, \tau_{0}\right)^{\mathrm{T}}$, which is the projection of period-one double-impact symmetrical orbit to the Poincaré section $\sigma$.

\section{Jacobian matrix of the transcendental map}

Eq. (2) is amenable to analytical treatment due to proportional damping. Between the $n$ and $(n+1)$ th impacts, the general solutions of Eq. (2) are give by

$$
\begin{aligned}
x_{i}(t)= & \sum_{j=1}^{2} \psi_{i j}\left(\mathrm{e}^{-\eta_{j} t}\left(a_{j n} \cos \omega_{d j} t+b_{j n} \sin \omega_{d j} t\right)+A_{j} \sin \left(\omega t+\tau_{n}\right)+B_{j} \cos \left(\omega t+\tau_{n}\right)\right) \\
\dot{x}_{i}(t)= & \sum_{j=1}^{2} \psi_{i j}\left(\mathrm{e}^{-\eta_{j} t}\left(\left(b_{j n} \omega_{d j}-\eta_{j} a_{j n}\right) \cos \omega_{d j} t-\left(\eta_{j} b_{j n}+a_{j n} \omega_{d j}\right) \sin \omega_{d j} t\right)\right. \\
& \left.+A_{j} \omega \cos \left(\omega t+\tau_{n}\right)-B_{j} \omega \sin \left(\omega t+\tau_{n}\right)\right),
\end{aligned}
$$

where $i=1,2, \omega_{j}(j=1,2)$ denote the eigenfrequencies of Eq. (2), $\psi_{i j}$ are the components of the canonical model matrix $\Psi$; modal damping ratio $\eta_{j}=\zeta \omega_{j}^{2}$, damping eigenfrequency $\omega_{d j}=\sqrt{\omega_{j}^{2}-\eta_{j}^{2}} ; a_{j n}$ and $b_{j n}$ are the constants of integration determined by model parameters and the state variables $X_{n}$ just after the $n$th impact, which are expressed by

$$
\begin{aligned}
a_{1 n} & =\frac{\psi_{22} x_{1 n}-\psi_{12} x_{2 n}}{\psi_{22} \psi_{11}-\psi_{12} \psi_{21}}-A_{1} \sin \tau_{n}-B_{1} \cos \tau_{n}, \\
a_{2 n} & =\frac{\psi_{21} x_{1 n}-\psi_{11} x_{2 n}}{\psi_{21} \psi_{12}-\psi_{11} \psi_{22}}-A_{2} \sin \tau_{n}-B_{2} \cos \tau_{n}, \\
b_{1 n} & =\left(\frac{\psi_{22} \dot{x}_{1 n+}-\psi_{12} \dot{x}_{2 n}}{\psi_{22} \psi_{11}-\psi_{12} \psi_{21}}+\eta_{1} a_{1 n}-A_{1} \omega \cos \tau_{n}+B_{1} \omega \sin \tau_{n}\right) / \omega_{d 1},
\end{aligned}
$$




$$
b_{2 n}=\left(\frac{\psi_{21} \dot{x}_{1 n+}-\psi_{11} \dot{x}_{2 n}}{\psi_{21} \psi_{12}-\psi_{11} \psi_{22}}+\eta_{2} a_{2 n}-A_{2} \omega \cos \tau_{n}+B_{2} \omega \sin \tau_{n}\right) / \omega_{d 2},
$$

where $x_{1 n}=\delta$ or $-\delta, X_{n}=\left(\dot{x}_{1 n+}, x_{2 n}, \dot{x}_{2 n}, \tau_{n}\right)^{\mathrm{T}}$ denote the state variables just after the impact occurring at the stop $A$ or $\bar{A}$.

Because of the existence of the impact, there is discontinuity in the velocity $\dot{x}_{1}$. Due to these impacts, we cannot use the algorithms usually applied to compute Lyapunov exponents in smooth systems. Thus, we introduce a transcendental map to develop an adapted algorithm that can be applied to the vibro-impact system described in this section. For the vibro-impact system, the discontinuities in the trajectory have to be supplemented with matching conditions at the impact instants. Such instants are determined by integrating the equations of motion in continuous time. The values of velocities and displacements of two masses and the phase shift at this time are taken as the dynamical variables of the transcendental map.

$$
\begin{aligned}
& \dot{x}_{1(n+1)+}=-r \sum_{j=1}^{2} \psi_{1 j}\left(\mathrm{e}^{-\eta_{j} t_{n+1}}\left(\left(b_{j n} \omega_{d j}-\eta_{j} a_{j n}\right) \cos \omega_{d j} t_{n+1}-\left(\eta_{j} b_{j n}+a_{j n} \omega_{d j}\right) \sin \omega_{d j} t_{n+1}\right)\right. \\
&\left.+A_{j} \omega \cos \left(\omega t_{n+1}+\tau_{n}\right)-B_{j} \omega \sin \left(\omega t_{n+1}+\tau_{n}\right)\right) \\
& x_{2(n+1)}= \sum_{j=1}^{2} \psi_{2 j}\left(\mathrm{e}^{-\eta_{j} t_{n+1}}\left(a_{j n} \cos \omega_{d j} t_{n+1}+b_{j n} \sin \omega_{d j} t_{n+1}\right)\right. \\
&\left.+A_{j} \sin \left(\omega t_{n+1}+\tau_{n}\right)+B_{j} \cos \left(\omega t_{n+1}+\tau_{n}\right)\right), \\
& \dot{x}_{2(n+1)=} \sum_{j=1}^{2} \psi_{2 j}\left(\mathrm{e}^{-\eta_{j} t_{n+1}}\left(\left(b_{j n} \omega_{d j}-\eta_{j} a_{j n}\right) \cos \omega_{d j} t_{n+1}-\left(\eta_{j} b_{j n}+a_{j n} \omega_{d j}\right) \sin \omega_{d j} t_{n+1}\right)\right. \\
&\left.+A_{j} \omega \cos \left(\omega t_{n+1}+\tau_{n}\right)-B_{j} \omega \sin \left(\omega t_{n+1}+\tau_{n}\right)\right), \\
& \tau_{n+1}= \begin{cases}\omega t_{n+1}+\tau_{n}-2 \pi, & \left(\omega t_{n+1}+\tau_{n} \geq 2 \pi\right) \\
\omega t_{n+1}+\tau_{n}, & \left(\omega t_{n+1}+\tau_{n}<2 \pi\right)\end{cases}
\end{aligned}
$$

where $x_{1(n+1)}=\delta$ or $-\delta$.

Using the map we can obtain the Jacobian matrix components necessary to calculate the Lyapunov exponents.

Using Eqs. (12)-(15) we develop the algorithm used to obtain the Lyapunov exponents. To calculate the Lyapunov exponents, we use the Jacobian matrix $J^{n}$ of the transcendental map

$$
J^{n}=\left[\begin{array}{cccc}
\frac{\partial \dot{x}_{1(n+1)+}}{\partial \dot{x}_{1 n+}} & \frac{\partial \dot{x}_{1(n+1)+}}{\partial x_{2 n}} & \frac{\partial \dot{x}_{1(n+1)+}}{\partial \dot{x}_{2 n}} & \frac{\partial \dot{x}_{1(n+1)+}}{\partial \tau_{n}} \\
\frac{\partial x_{2(n+1)}}{\partial \dot{x}_{1 n+}} & \frac{\partial x_{2(n+1)}}{\partial x_{2 n}} & \frac{\partial x_{2(n+1)}}{\partial \dot{x}_{2 n}} & \frac{\partial x_{2(n+1)}}{\partial \tau_{n}} \\
\frac{\partial \dot{x}_{2(n+1)}}{\partial \dot{x}_{1 n+}} & \frac{\partial \dot{x}_{2(n+1)}}{\partial x_{2 n}} & \frac{\partial \dot{x}_{2(n+1)}}{\partial \dot{x}_{2 n}} & \frac{\partial \dot{x}_{2(n+1)}}{\partial \tau_{n}} \\
\frac{\partial \tau_{n+1}}{\partial \dot{x}_{1 n+}} & \frac{\partial \tau_{n+1}}{\partial x_{2 n}} & \frac{\partial \tau_{n+1}}{\partial \dot{x}_{2 n}} & \frac{\partial \tau_{n+1}}{\partial \tau_{n}}
\end{array}\right] .
$$

The derivation of the components in matrix (16) is introduced in the Appendix.

\section{Lyapunov exponents}

The computation algorithm is described to calculate the Lyapunov exponents in vibro-impact systems using a transcendental map in Ref. [29]. Consider $m$-dimensional discrete dynamical system

$$
X_{k+1}=F\left(X_{k}\right), \quad X_{k} \in \boldsymbol{R}^{m} .
$$

The Lyapunov exponents are expressed by

$$
\lambda_{j}=\lim _{N \rightarrow \infty} \frac{1}{N} \ln \left|\Lambda_{j}^{N}\right|, \quad j=1,2, \ldots \ldots, m,
$$




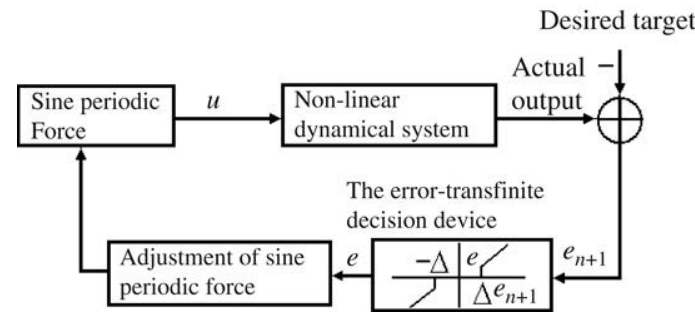

Fig. 2. Schematic of the method for suppressing chaos by using sine periodic force.

where $\left|\boldsymbol{\Lambda}_{j}^{N}\right|$ are eigenvalues of the Jacobian matrix $M$ given by

$$
M=\prod_{n=1}^{N} J^{n}
$$

where $J^{n}$ is the Jacobian matrix of the $n$th iteration (impact) of the transcendental map.

As have been mentioned in Ref. [29], Eq. (18) cannot be used in the computation algorithm due to the matrix $M$ obtained by the product (19). Even for a few iterations, the components of matrix $M$ become very large for chaotic attractors and null for the periodic attractors so that the numerical calculation becomes impracticable. To eliminate this problem, a way is provided to convert the matrix $M$ into a product of the orthogonal matrices and the upper right triangular matrices [29], whose eigenvalues are the numbers along the diagonal and the Lyapunov exponents can be expressed by

$$
\lambda_{j}=\lim _{N \rightarrow \infty} \frac{1}{N} \sum_{n=1}^{N} \ln \left|a_{j}^{n}\right|, \quad j=1,2, \ldots \ldots m,
$$

where $a_{j}^{n}$ are diagonal components of $n$th iteration of the product of the upper right triangular matrices. Thus, the Lyapunov exponents are obtained from a sum in (20) instead of a product.

Furthermore, we cannot perform exactly the infinite sum in (20) for numerical calculations, but the convergence of the Lyapunov exponents occurs with good precision after just a few thousand iterations, both for periodic and chaotic attractors.

\section{Suppressing chaos in the vibro-impact systems}

\subsection{Suppressing chaos in the vibro-impact systems by sine periodic force}

Sine periodic force is a driving one which is easy to apply and control. Zhao and Wang [41] presented a method of controlling bifurcation and chaos by adjusting the external driving force. The method is proved to be effective by the presentation of examples of suppressing bifurcation and chaos for an impact damper. Chaotic impacts of vibratory systems with a clearance are suppressed by applying the controlling method in the paper. The schematic of the method for suppressing chaos by using sine periodic force is shown in Fig. 2 [41]. The desired target is some measurable state variable while the system is in a stable state, as for example, impact period, displacements or velocities, etc. The desired target is preserved as an ideal output. The practical output in Fig. 2 is the real time test value of measurable state variable. $e_{n+1}$ is the error between the practical target and the desired target at $n+1$ time. $u$ is the external driving force. The external sine driving force is expressed by

$$
u=B \sin (\gamma \omega t),
$$

where $B$ and $\gamma$ are adjustable parameters controlled by the adjustment algorithm of external driving force.

The error-transfinite decision device satisfies the relationships: when $\left|e_{n+1}\right|<\Delta, e=0$, there is no control; when $\left|e_{n+1}\right|>\Delta, e=e_{n+1}$, the control is applied. The symbol $\Delta$ represents the threshold value of allowable deviation of the system.

For Fig. 2, if the error between the practical target and the desired target satisfies the condition $\left|e_{n+1}\right|<\Delta, e=0$, the adjustment algorithm makes the value of $u$ equal zero. As a result, the external driving force is not applied. If 
$\left|e_{n+1}\right|>\Delta, e=e_{n+1}$ is produced by the adjustment algorithm of external driving force so that its parameters, $B$ and $\gamma$, are dynamically adjusted until the system is in a stable state, i.e., $e=0$.

To this end, the adjustable parameters $B$ and $\gamma$ of driving signal $u=B \sin (\gamma \omega t)$ must be adjusted with increase of $n$ until the value of $\left|e_{n+1} / e_{n}\right|$, from larger than 1 to less than 1 , is changed. Consequently, $\left|e_{n+1}\right|$ finally becomes less than the threshold value $\Delta$ of the error-transfinite decision device.

For harmonically excited systems with impacts, one can usually take $\gamma=1$ so as to make the external excitation frequency equal the vibrating frequency of the original system. The practical move to fulfill the control is stated in the ensuing paragraphs.

(1) the initial value $B_{0}=0$.

(2) if $\left|e_{n+1}\right|<\Delta$ at $n+1$ time, no external driving force is applied due to $e=0, B_{n+1}=B_{0}=0$ and $u=0$ in Fig. 2 so that the next decision returns to the (2) cycle again; if $\left|e_{n+1}\right| \geq \Delta$, the decision $e=e_{n+1}$ brings out an external driving force, and diverts to the (3) process.

(3) $u$ is created by the adjustment algorithm of external driving force and expressed by

$$
B_{n+1}=B_{n}+\alpha\left|e_{n+1}\right|, \quad u=B_{n+1} \sin (\gamma \omega t),
$$

where $\alpha$ is a weight factor, and $0<\alpha \leq 1$.

(4) After the driving force is applied, if $\left|e_{n+1}\right| \geq \Delta$, the adjustment direction of $B$ is modified according to the value of $\left|e_{n+1} / e_{n}\right|$. If $\left|e_{n+1} / e_{n}\right|>1, B$ is adjusted in the contrary direction so that let $\alpha=-\alpha$. Whereas, $\alpha$ is fixed and the (3) process is continued. If new $\left|e_{n+1}\right|<\Delta$, the error-transfinite decision device brings out the output of $e=0$, the coefficient adjustment of driving force ends. The final coefficient is expressed by $B^{*}$, and the external driving force is given by

$$
u=B^{*} \sin (\gamma \omega t),
$$

This final adjustment brings into effect that the chaos are suppressed by applying a sine periodic force with the amplitude $B^{*}$ to the system.

\subsection{Suppressing chaos in the vibro-impact systems using period pulse}

Consider a general nonlinear mapping system

$$
X_{n+1}=F\left(X_{n}\right),
$$

where $X \in \boldsymbol{R}^{m}$.

Starting from an initial point $X_{0}$, after $p$ th iteration, the system (24) can be written as

$$
X=F^{(P)}\left(X_{0}\right) .
$$

The convergence property after $p$ th iteration from the initial points can be known from the Jacobian matrix of the map (25). The stability condition of periodic $P$ orbit is all moduli of eigenvalues of Jacobian matrix are less than 1 . Assuming that the system exits in stable period- $P$ orbit, from Eq. (25), one knows that $X_{P}=X_{0}$. If the variation of the system parameters causes bifurcation or chaos of the system, the map will deviate from the original stable fixed points. That is $X_{P} \neq X_{0}$.

The idea of the control method is to present the impulse signal $Q$ to the system at the $P$ period point [41],

$$
Q=X_{P}-X_{0} .
$$

Thus, the orbit, which starts from an initial point $X_{0}$, after $p$ th iteration combined with impulse signal $Q$, will be

$$
F^{(P)}\left(X_{0}\right)-Q=X_{0} \text {. }
$$

When $X_{P}=X_{0}, Q=0$ there is no control. When $X_{P} \neq X_{0}, Q \neq 0$ will counteract departure. Then the system maps continuously according to Eq. (25). Repeating the above process, it is equal to presenting a period pulse to the system. The period pulse can be written by

$$
Q_{n}=\sum_{k=0} Q \delta(n-k P) .
$$

$k=0$ is the moment when the control is applied. 


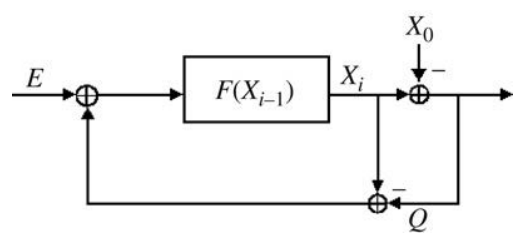

Fig. 3. Schematic of the method of periodic impulse suppressing chaos.

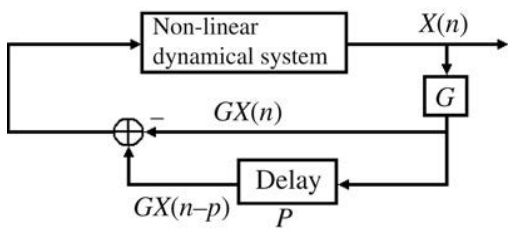

Fig. 4. Schematic of principle of the time delay feedback controlling.

Impulse signal $Q$ makes the orbit end-to-end, thus forms disturbed periodic orbit $X_{0}, X_{1}+\Delta X_{1}, X_{2}+$ $\Delta X_{2}, \ldots, X_{P-1}+\Delta X_{P-1}$ and suppresses the chaos eventually. The purpose of this method is not to make the control system return to the original stable periodic orbit but to make it to be in periodic motion all the time so as to suppress the chaos.

As for the vibro-impact system with a clearance, we take one reciprocating impaction as a period, i.e., $P=1$. When the system is stable, it satisfies

$$
X_{0}=F\left(X_{0}\right) .
$$

The schematic of the method of periodic impulse suppressing chaos is illustrated in Fig. 3 [41]. $X_{i}$ denotes the input at $i$ moment and $X_{0}$ denotes the stable output vector (control target). $E$ denotes noise from measurement error, time delay error, and other factors. $X_{i}$ is detected in real time. If $X_{i}=X_{0}$, then $Q=0$, no control is applied; if $X_{i} \neq X_{0}$, then $Q=\Delta X_{i}$, where $\Delta X_{i}$ is the derivation of $X_{i}$ from $X_{0}$ and the error $\Delta X_{i}$ is used as the impulse control signal.

\subsection{Suppressing chaos of the vibro-impact systems based on delay feedback}

The controlling method is to skillfully use certain parts of output signals of the system, through time delaying and subtracting it with original output signals, to get the controlling information to be fed back to the system.

The time delay feedback can be given as the following dynamic forms

$$
X(n+1)=F(X(n))+G(X(n-p)-X(n)),
$$

where, $X \in R^{m}, G=\operatorname{Diag}\left[g_{1}, g_{2}, \ldots, g_{i}, \ldots, g_{m}\right], g_{1}=g_{2}=\cdots=g_{i}(i \leq m)$ are the feedback ratio factors.

The Fig. 4 shows the schematic of principle of the time delay feedback controlling. When $G=0$, the original system exhibits chaotic motion. $X(n)$ and $X(n-p)$ are the measurements of $X$ at the time $n$ and the time $n-p$, respectively. The period of certain unstable period motion can be chosen as the delay time $p$. When the feedback matrix $G$ has been properly chosen, the system would be driven to the expected orbit.

\section{Dynamic analysis of the vibratory system with a clearance}

Numerical analyses are carried out for determining the dynamical responses of the vibro-impact system. According to the non-dimensional parameters and variables (1), we have the ranges of these parameters $\mu_{m}, \mu_{k}$ and $\mu_{c}$ as $\mu_{m} \in(0,1), \mu_{k} \in(0,1)$ and $\mu_{c} \in(0,1)$. The mid-values of parameters $\mu_{m}, \mu_{k}$ and $\mu_{c}$ are considered. The system parameters: $\mu_{m}=0.5, \mu_{k}=0.5, \mu_{c}=0.5, \zeta=0.1, r=0.8$ and $\delta=0.1-0.6$ are chosen for analyses. Clearance is the most important system parameter for the nonlinear system shown in Fig. 1. The influence of parameters $\omega$ and $\delta$ on symmetrical double-impact periodic motion and bifurcations is depicted by the bifurcation diagram shown in Fig. 5. Representative bifurcation diagrams of before-impact velocity and the corresponding Lyapunov exponents as a function of the excitation frequency $\omega$ are shown for $\delta=0.1,0.3,0.545$ and 0.6 in Fig. 6(a)-(d) and (a1)-(d1), 


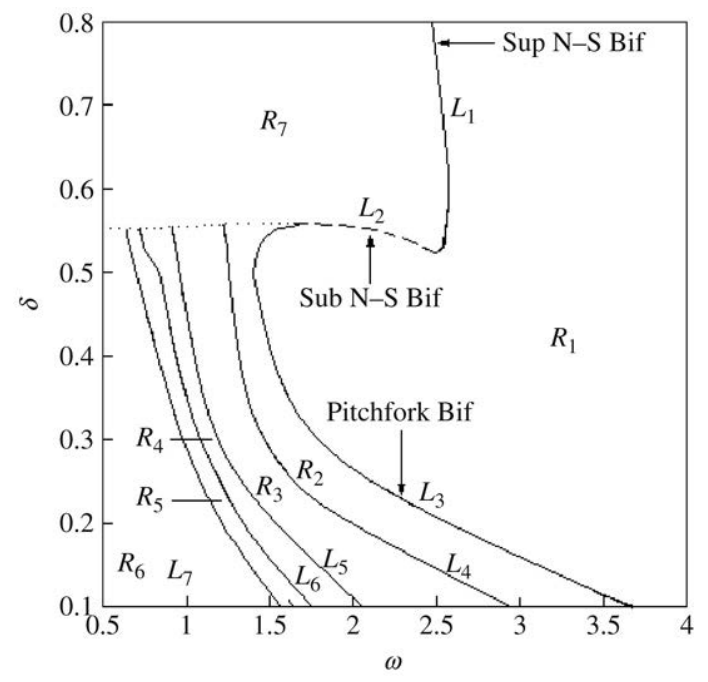

Fig. 5. Bifurcation diagram associated with parameters $(\omega, \delta)$, system parameters: $\mu_{m}=0.5, \mu_{k}=0.5, \mu_{c}=0.5, \zeta=0.1, r=0.8$.

respectively, which illustrate the bifurcation characteristics of 1-1-1 symmetrical motion. The system exhibits stable 1-1-1 symmetrical motion in the region $R_{1}$ of Fig. 5. It undergoes a supercritical Neimark-Sacker bifurcation on the line $L_{1}$, giving rise to a family of attracting invariant circles associated with the fixed point of 1-1-1 symmetrical motion, and a subcritical Neimark-Sacker bifurcation on the line $L_{2}$. There exist quasi-periodic impact motions associated with 1-1-1 symmetrical one, long-period multi-impact motions and chaotic motions in the region $R_{7}$. Crossing the curve $L_{3}$ from the region $R_{1}$ to the region $R_{2}$, pitchfork bifurcation of 1-1-1 symmetrical motion occurs, and 1-1-1 unsymmetrical motion stabilizes in the region $R_{2}$. Crossing the curve $L_{4}$ from the region $R_{2}$ to the region $R_{3}$, Neimark-Sacker bifurcation generates a family of invariant circles associated with the fixed point of 1-1-1 unsymmetrical motion. In the region $R_{3}$, the system exhibits quasi-periodic impact motions associated with 1-1-1 unsymmetrical one, long-period multi-impact motions and chaotic motions, etc. Crossing the curve $L_{5}$ from the region $R_{3}$ to the region $R_{4}$ stable 1-2-2 symmetrical motion is generated by a degeneration of chaos. Crossing the curve $L_{6}$ from the region $R_{4}$ to the region $R_{5}$, pitchfork bifurcation of 1-2-2 symmetrical motion occurs, and 1-2-2 unsymmetrical motion stabilizes. In the region $R_{5}$, the 1-2-2 unsymmetrical motion is of two antisymmetrical forms due to different initial conditions and symmetry of the structure and excitation point. Crossing the curve $L_{7}$, period-doubling bifurcation of 1-2-2 unsymmetrical motion occurs. Thereafter, the motion undergoes a succession of period-doubling bifurcations with decrease in $\omega$, which eventually result in apparently non-periodic or chaotic motions. For smaller values of clearance $\delta, 1-1-1$ symmetrical motion usually undergoes pitchfork bifurcation, and smaller values of clearance generally lead to the enlarged areas of chaotic motions, see Figs. 5 and 6(a). For a relatively narrow range of clearance value $\delta \in[0.5352,0.5602], 1-1-1$ symmetrical motion undergoes supercritical Neimark-Sacker bifurcation, advise subcritical Neimark-Sacker bifurcation and pitchfork bifurcation in turn with decrease in the forcing frequency $\omega$; see Figs. 5 and 6(c). For large values of clearance $\delta, 1-1-1$ symmetrical motion undergoes supercritical Neimark-Sacker bifurcation with decrease in the forcing frequency $\omega$; see Figs. 5 and 6(d). However, for larger values of $\delta$ the mass $M_{1}$ cannot hit the stops, and the system will undergo simple oscillations and behave as a linear system.

The detailed simulative results are presented in the form of phase plane portraits, time series and projected portraits of Poincaré map for the vibratory system with a clearance $\delta=0.1$. By looking at the bifurcation diagram in Fig. 6(a), we have a large window of 1-1-1 symmetrical motion for $\omega$ in the interval $\omega \in[3.6907,5]$. With the parameter $\omega$ reducing, pitchfork bifurcation of 1-1-1 symmetrical motion occurs at $\omega=3.6907$. Consequently, 1-1-1 symmetrical motion becomes 1-1-1 unsymmetrical one which is of two antisymmetrical forms due to different initial conditions and symmetrical stops. Thereafter, the unsymmetrical double-impact periodic motions undergo Neimark-Sacker bifurcation with further decrease in $\omega$. We give an example of a 1-1-1 symmetrical motion which exists at a forcing frequency of $\omega=3.7$. A phase portrait of this periodic motion is shown in Fig. 7(a), time series in Fig. 7(b). A phase portrait of 1-1-1 unsymmetrical motions is shown for $\omega=3.5$ in Fig. 8(a), in which two 1-1-1 antisymmetrical 

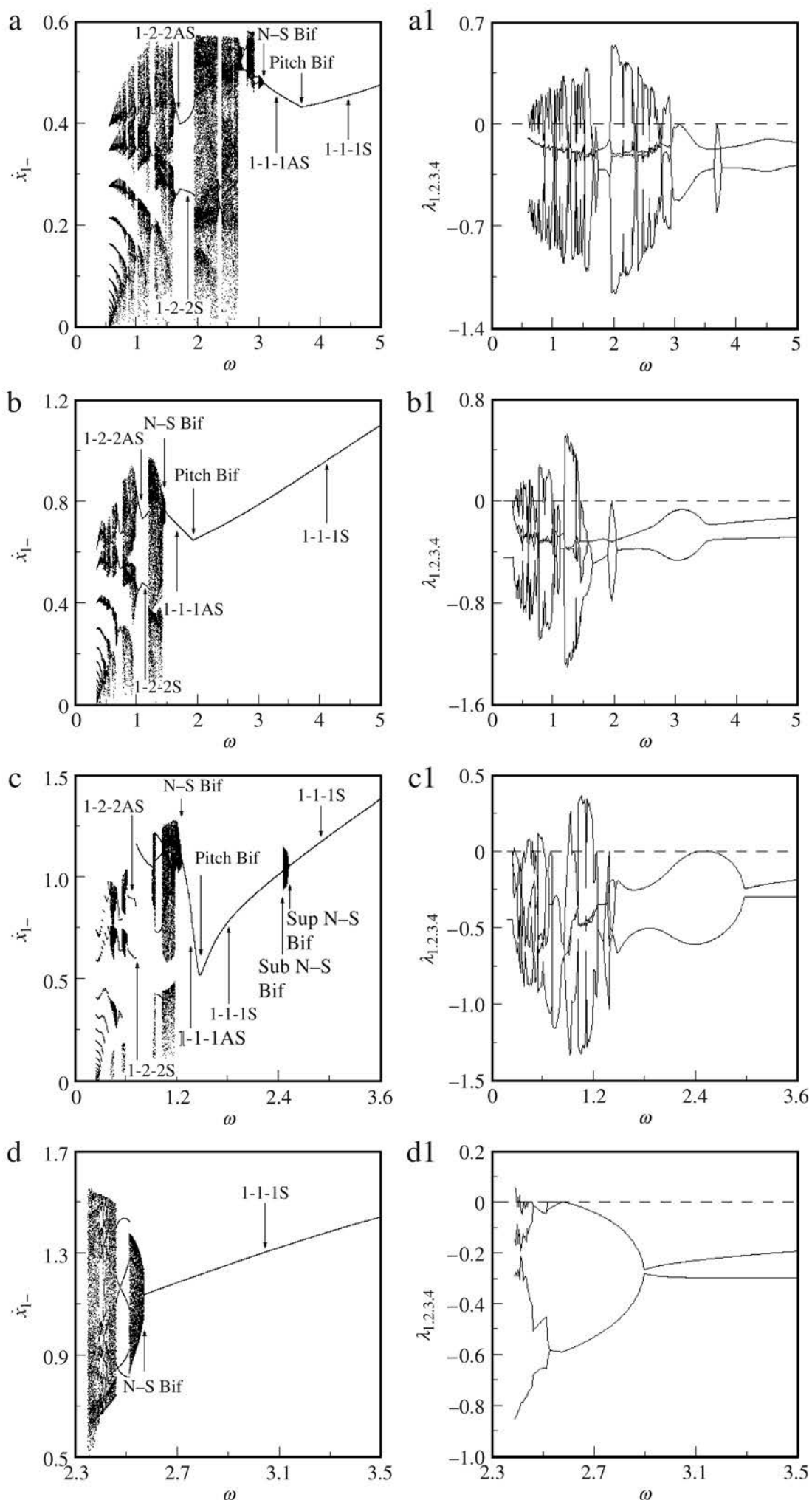

Fig. 6. Bifurcation diagrams of before-impact velocity and corresponding Lyapunov exponents as a function of the excitation frequency $\omega$ : (a) and (a1) $\delta=0.1$; (b) and (b1) $\delta=0.3$; (c) and (c1) $\delta=0.545$; (d) and (d1) $\delta=0.6$ 

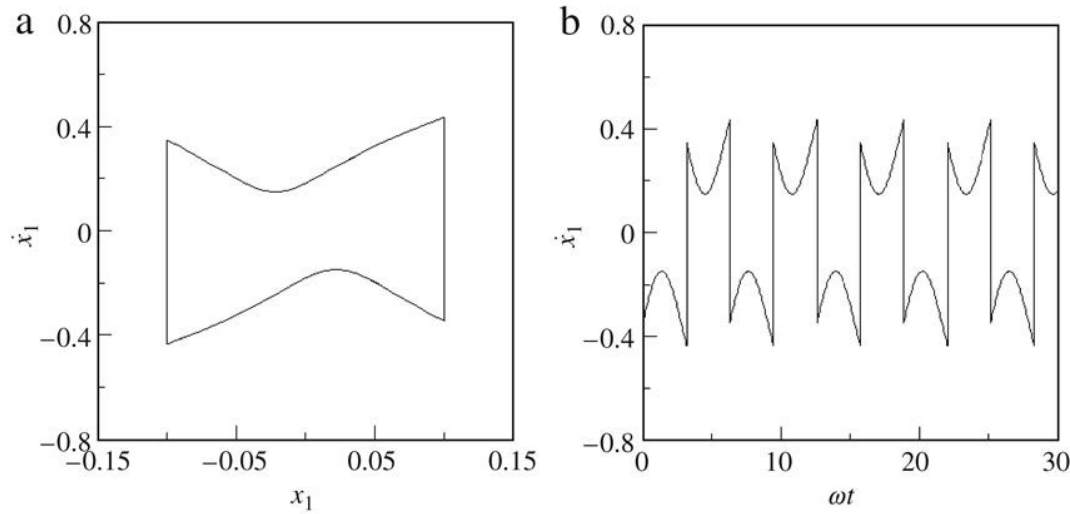

Fig. 7. Phase plane portrait and time series: 1-1-1 symmetrical motion, $\delta=0.1, \omega=3.7$.
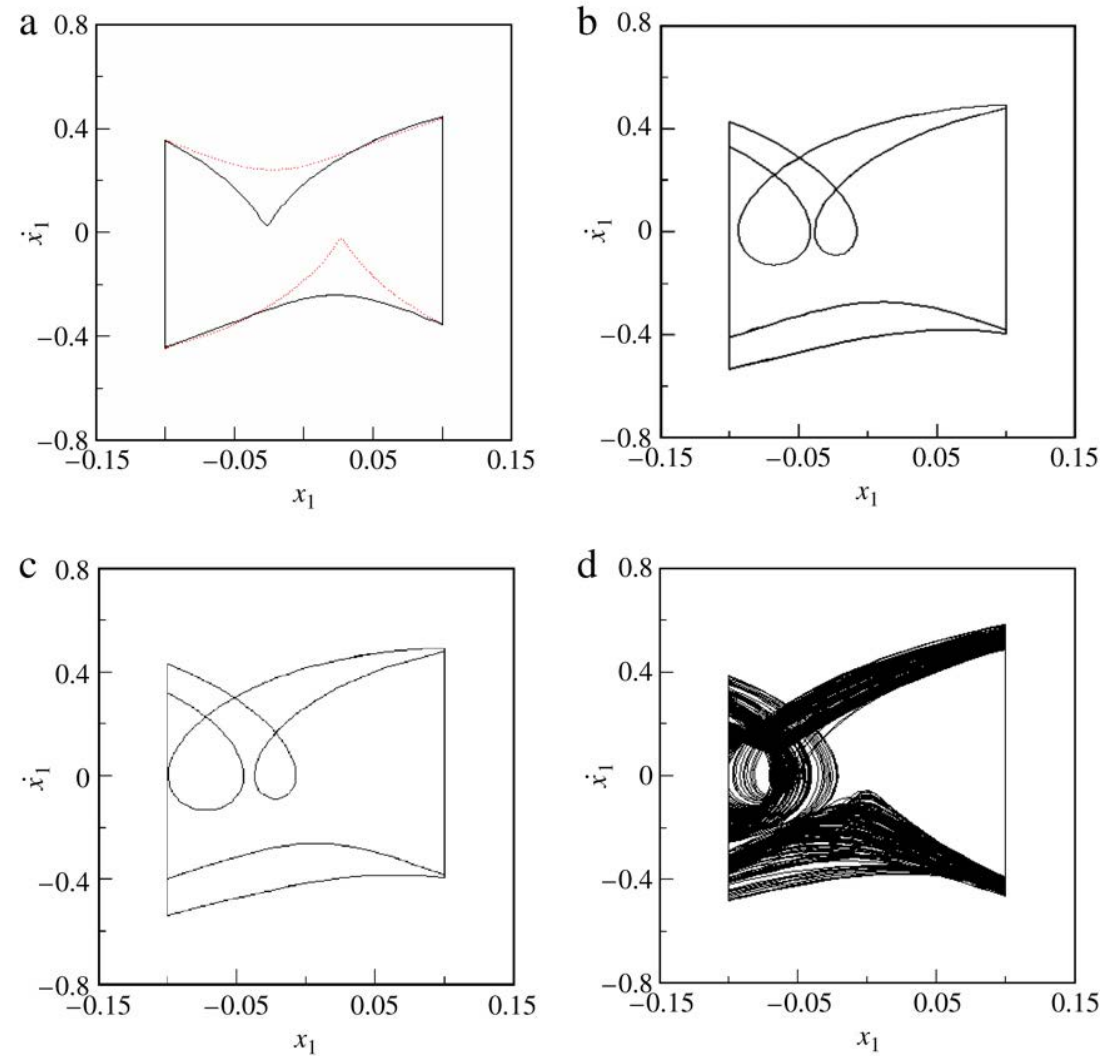

Fig. 8. Phase plane portraits: (a) 1-1-1 unsymmetrical motion, $\delta=0.1, \omega=3.5$; (b) 2-2-2 motion, $\delta=0.1, \omega=2.95$; (c) $2-2-2$ motion with grazing a stop, $\delta=0.1, \omega=2.93193 ;$ (d) chaotic motion, $\delta=0.1, \omega=2.83$.

orbits, caused by different initial conditions, are represented respectively by real lines and dotted lines. The projected Poincaré section, near the value of Neimark-Sacker bifurcation, are shown in Fig. 9. Transient points as well as the quasi-periodic attractor associated with 1-1-1 unsymmetrical point, starting from the initial condition near the unstable fixed point of 1-1-1 symmetrical motion, are shown in Fig. 9(a). With further decrease in $\omega$, phase locking takes place so that the quasi-periodic impact motion gets locked into a period-two attractor (see Figs. 8(b) and 9(b)). As the forcing frequency $\omega$ passes through $\omega=2.93193$ decreasingly, a grazing contact instability of 2-2-2 motion occurs as seen in Fig. 8(c). Consequently, one impact in the motion period will increase and the 2-2-2 motion transits 

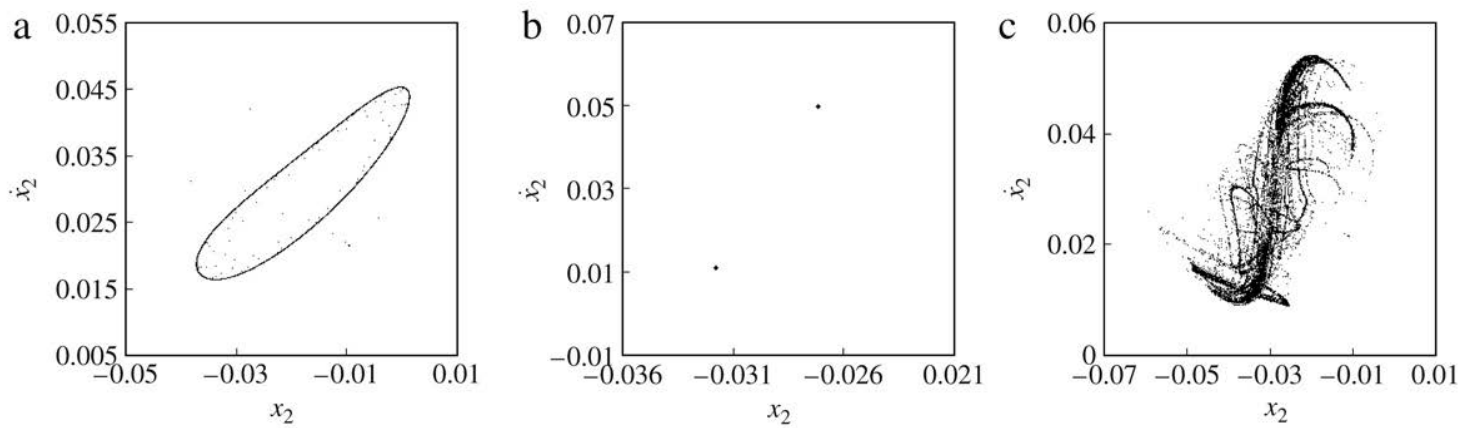

Fig. 9. Projected Poincaré sections $(\delta=0.1)$ : (a) quasi-periodic attractor associated with the fixed point of 1-1-1 unsymmetrical motion, $\omega=3.03$; (b) fixed points of 2-2-2 motion, $\omega=2.95$; (c) chaos, $\omega=2.83$.
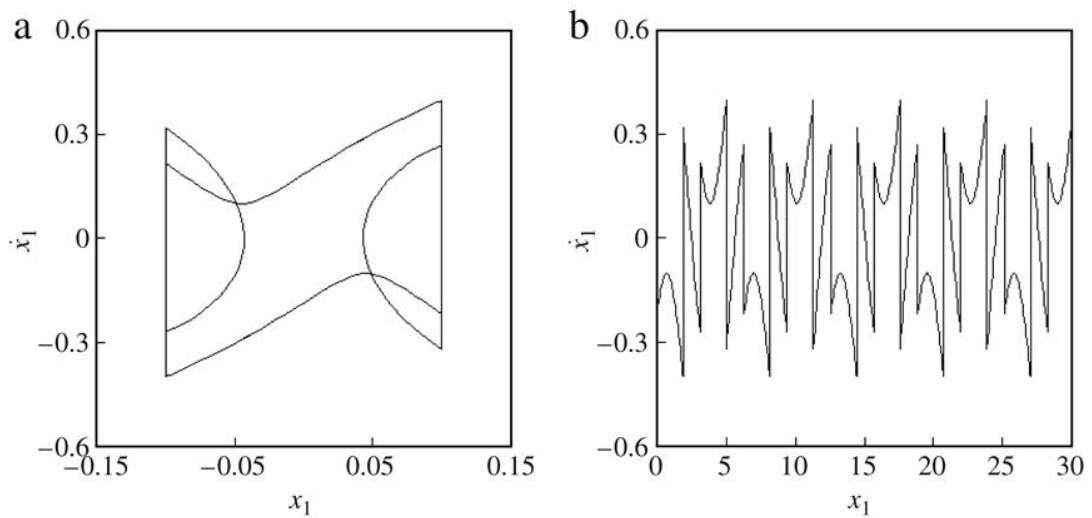

Fig. 10. Phase plane portrait and time series: 1-2-2 symmetrical motion, $\delta=0.1, \omega=1.72$.
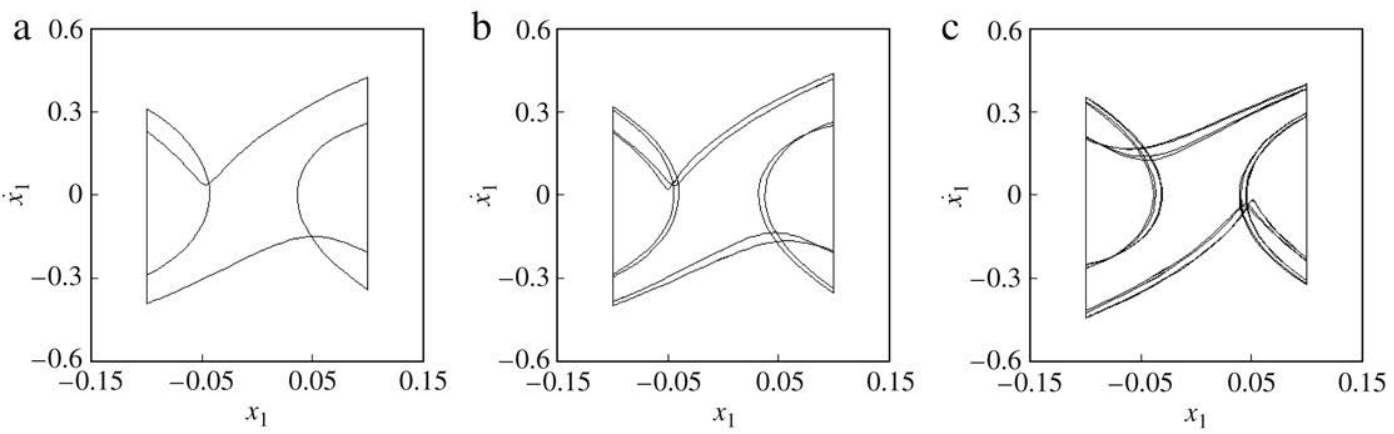

Fig. 11. Phase plane portraits $(\delta=0.1)$ : (a) 1-2-2 unsymmetrical motion, $\omega=1.65$; (b) 2-4-4 unsymmetrical motion, $\omega=1.63$; (c) $4-8-8$ unsymmetrical motion, $\omega=1.628$.

into chaotic motion via grazing bifurcation; see Figs. 8(d) and 9(c). Looking at the bifurcation diagram in Fig. 6(a), we have a large window of 1-1-1 symmetrical motion for $\omega$ in the interval $\omega \in[3.6907,5]$, a window of 1-1-1 unsymmetrical motion for $\omega \in[3.0738,3.6907]$. Two windows of 1-2-2 symmetric and unsymmetric motions are also observed from Fig. 6(a), respectively. 1-2-2 symmetrical motion is generated by a degeneration of chaos with decrease in $\omega$. We give an example of a 1-2-2 symmetrical motion which exists at a forcing frequency of $\omega=1.72$. A phase portrait of this periodic motion is shown in Fig. 10(a), time series in Fig. 10(b). Pitchfork bifurcation of 1-2-2 symmetrical motion occurs as $\omega$ passes through $\omega=1.7031$ decreasingly. Consequently, 1-2-2 unsymmetrical motion is created. A phase portrait of 1-2-2 unsymmetrical motion is shown for $\omega=1.65$ in Fig. 11(a). With further decrease in $\omega$, the system falls into chaotic-impact motion via period-doubling sequences of 1-2-2 unsymmetrical motion. 

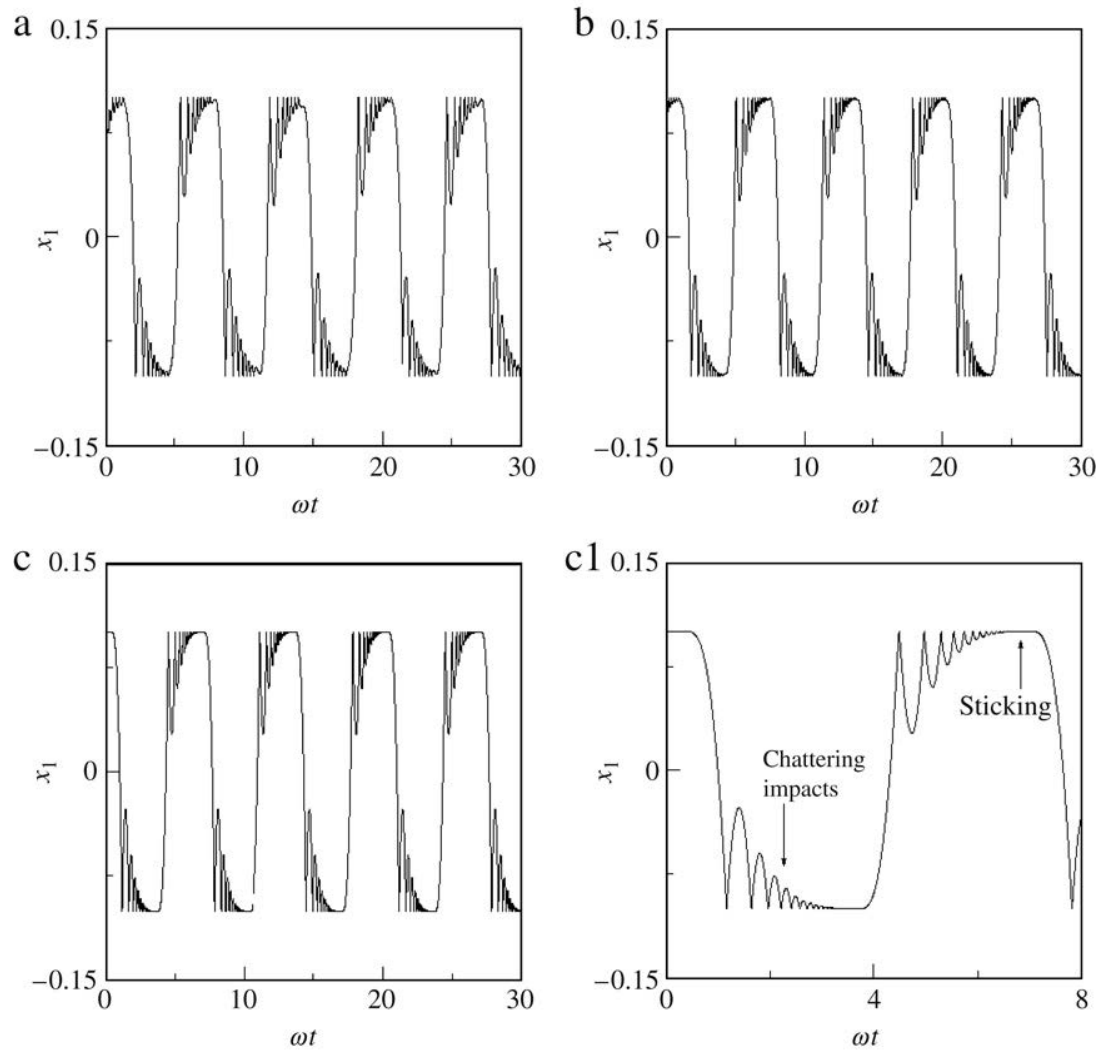

Fig. 12. Time series with chattering-impact vibration $(\delta=0.1)$ : (a) non-periodic chattering-impact vibration without sticking, $\omega=0.65$; (b) periodic chattering-impact vibration without sticking, $\omega=0.6$; (c) chattering-impact vibration with sticking, $\omega=0.57$; (c1) local zooming of figure (c).

Fig. 11 shows an important sequence of bifurcations, where the system bifurcates from period one (Fig. 11(a)) to period two (Fig. 11(b)), then from period two to period four (Fig. 11(c)). The chattering-impact motions are found to exist in the region of low forcing frequency. With decrease in $\omega$, non-periodic chattering-impact vibration transits to periodic one. Thereafter, sliding bifurcation of periodic chattering-impact vibration occurs. Consequently, periodic chattering-impact vibration with sticking is created. These imply such motions are very complex, varying from nonsticking types of chattering-impact vibration to sticking types of chattering-impact motion. Time series of non-sticking chattering-impact solutions are shown for $\omega=0.65$ and 0.6 in Fig. 12(a) and (b), respectively. A time series of sticking chattering-impact vibration is shown for $\omega=0.57$ in Fig. 12(c), a local zooming of Fig. 12(c) is shown in Fig. 12(c1). This sticking chattering-impact vibration is periodic, which includes regions of free flight, chattering and sticking for the mass $M_{1}$. Sticking in impact systems is mathematically analogous to sliding in electrical systems [59]. The sliding orbits in these electrical systems have been shown to exhibit particular types of sliding bifurcations under parameter variation [60]. The sliding dynamics on the separation boundary is discussed based on the set-valued vector field theory and the switching bifurcation conditions from the passable boundary to the non-passable boundary are developed in Ref. [61]. The normal vector product field function is introduced to determine the switching bifurcation and sliding fragmentation in [61]. It is important to note that chattering-impact vibration enlarges the adverse effects such as high noise levels, wear and tear and so on. For $\delta=0.545,1-1-1$ symmetrical motion undergoes supercritical Neimark-Sacker bifurcation, advise subcritical Neimark-Sacker bifurcation and pitchfork bifurcation in turn with decrease in the forcing frequency $\omega$; see Fig. 6(c). The sticking and non-sticking types of chattering-impact motions are also found to occur in the region of low forcing frequency; see Fig. 13(a)-(c). It is observed obviously, from Fig. 6(d), that period-one double-impact symmetrical motion of the vibro-impact system undergoes Neimark-Sacker bifurcation, and chaotic-impact motion can occur via phase locking with decrease in the forcing frequency $\omega$. The phase plane portrait and projected Poincaré section near the value of Neimark-Sacker bifurcation are shown in 

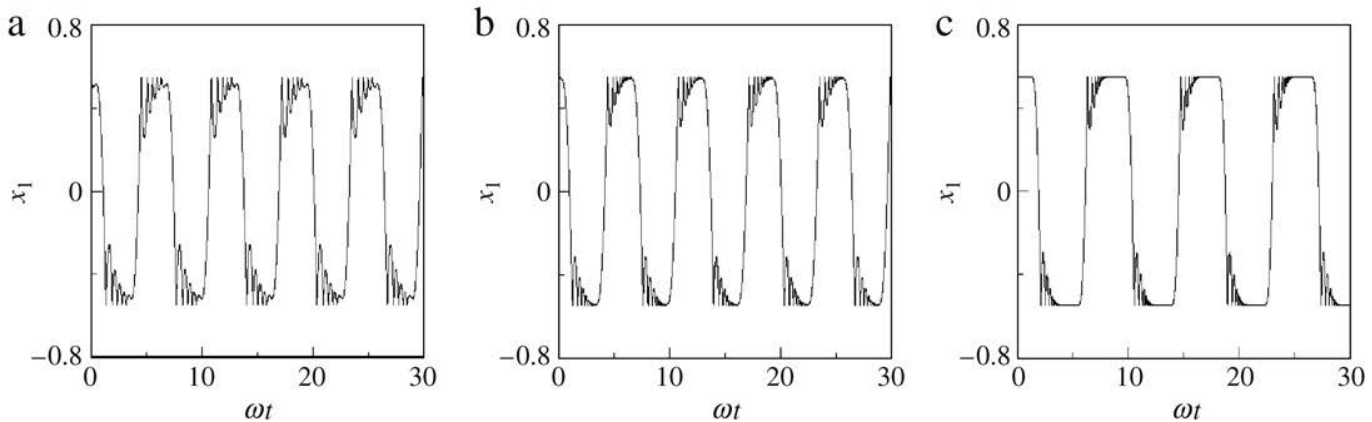

Fig. 13. Time series with chattering-impact vibration $(\delta=0.545$ ): (a) chattering-impact vibration without sticking, $\omega=0.35$; (b) chattering-impact vibration without sticking, $\omega=0.3$; (c) chattering-impact vibration with sticking, $\omega=0.25$.
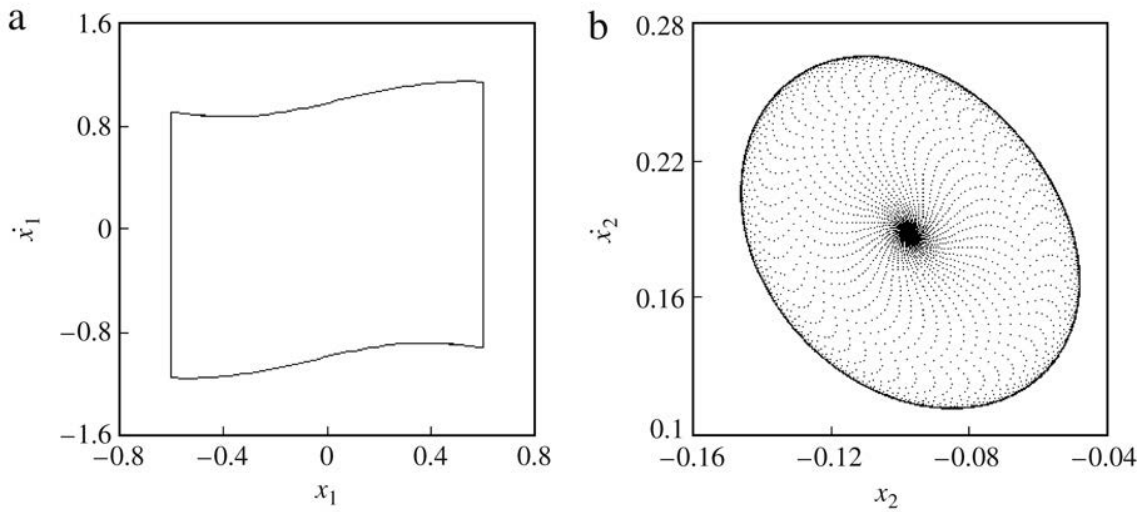

Fig. 14. Phase plane portrait and projected Poincaré section: (a) phase plane portrait of 1-1-1 symmetrical motion, $\delta=0.6, \omega=2.582 ;$ (b) attracting invariant circle associated with the fixed point of 1-1-1 symmetrical motion, $\delta=0.6, \omega=2.57$.

Fig. 14(a) and (b), respectively. The phase plane portrait of period-one double-impact symmetrical motion is plotted for $\omega=2.582$ in Fig. 14(a), the periodic-impact motion is represented by a stable fixed point in the projected Poincaré section. The attracting invariant circle associated with the fixed point of 1-1-1 symmetrical motion, in the projected Poincaré section, is shown for $\omega=2.57$ in Fig. 14(b).

\section{Suppressing chaotic impacts of the vibratory system with a clearance}

\subsection{Suppressing chaotic impacts by adjusting the driving force}

Suppressing chaotic impacts by using sine periodic force is stated in Section 6. The system with non-dimensional parameters: $\mu_{m}=0.5, \mu_{k}=0.5, \mu_{c}=0.5, \zeta=0.1$ and $r=0.8$ are still chosen for analysis. The method effectiveness is analyzed by the presentation of example for the impact oscillator with a clearance $\delta=0.1$. Bifurcation diagram of before-impact velocity and Lyapunov exponents as a function of the excitation frequency $\omega$ are shown for $\delta=0.1$ in Fig. 6(a) and (a1), respectively. The detailed analysis is presented, which has been illustrated by Figs. 7-12. The system exhibits 1-1-1 symmetrical motion at $\omega=3.7$ and chaotic-impact motion at $\omega=2.83$, as seen in Figs. 7(a), 8(d) and 9(c), respectively. The fixed point $X_{0}=\left(\dot{x}_{1+}, x_{2}, \dot{x}_{2}, \tau_{0}\right)=$ $(0.34545790,-0.00634090,0.02116031,1.80213070)^{\mathrm{T}}$ of $1-1-1$ symmetrical motion at $\omega=3.7$ is taken as the desired fixed point. The impact period and the time interval between consecutive impacts occurring at the same stop are easy to measure in practical engineering. Consequently, the angle shift is simple to determine. Based on the consideration, we choose the variable $\tau_{0}$ (phase shift) in the Poincaré section as the desired target. We take the phase shift $\tau$ as the practical output, and $\alpha=0.327, \Delta=10^{-5}$. Fig. 15 shows the simulative results after applying 

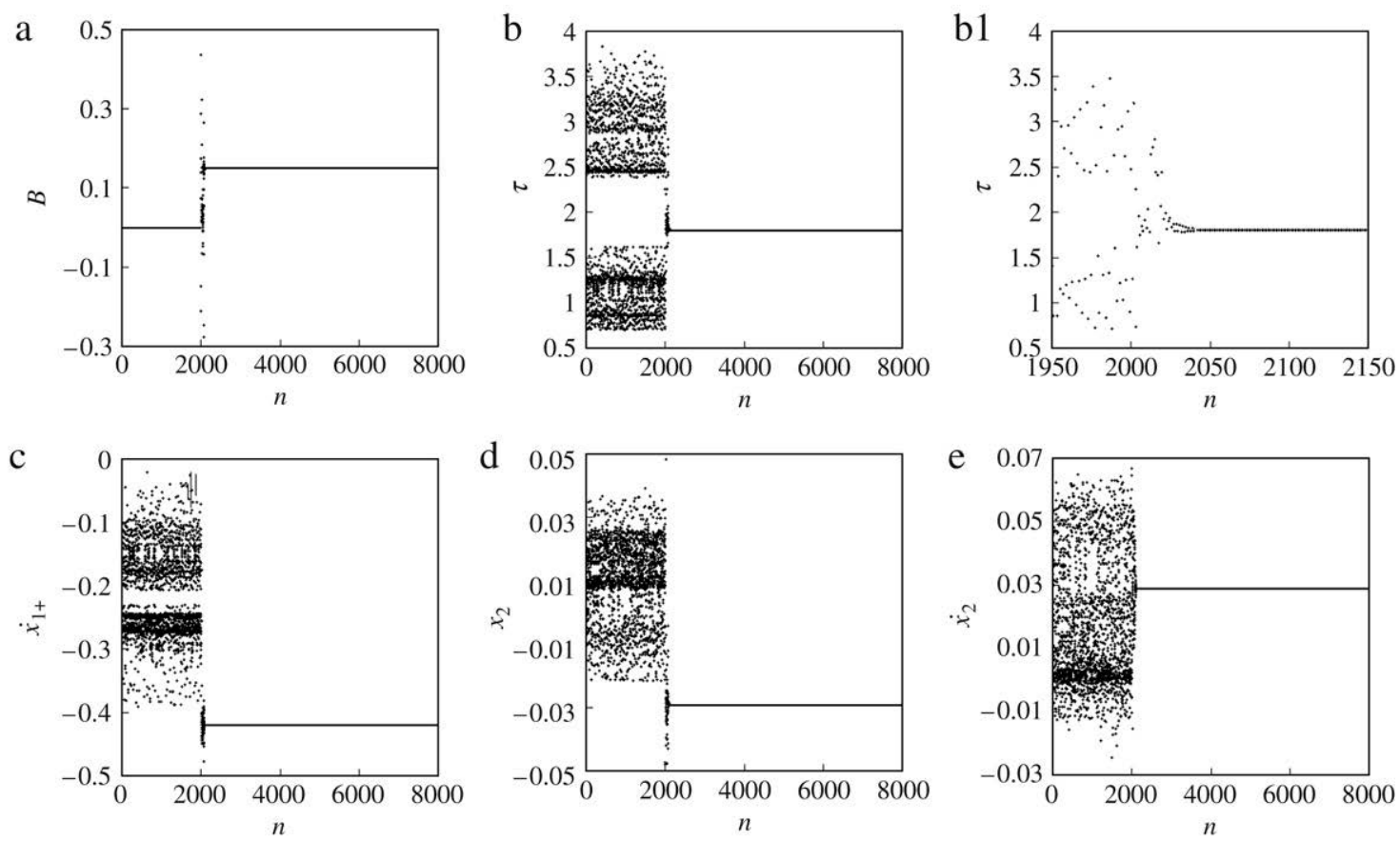

Fig. 15. Time responses of some variables (no controlling $(n<2000)$, after controlling $(n \geq 2000)$ ), $\omega=2.83$.
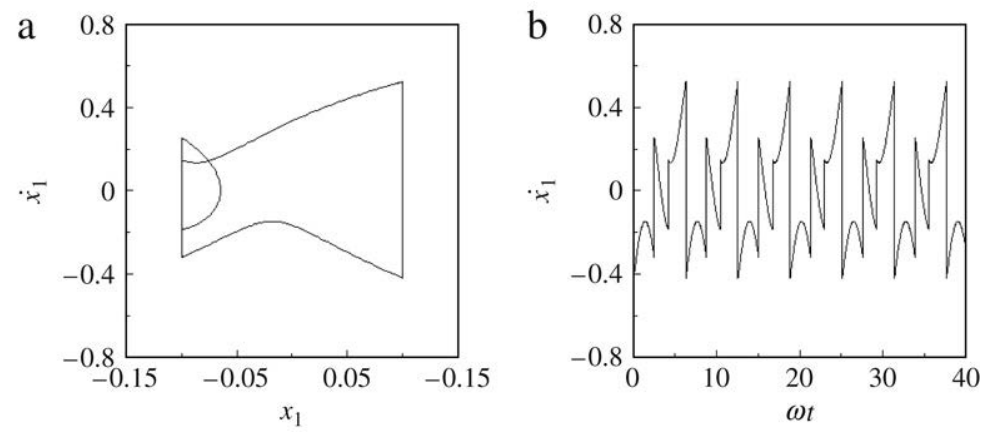

Fig. 16. Phase plane portrait and time series after controlling, $\omega=2.83$.

the control on the chaotic-impact motion which exists at a forcing frequency of $\omega=2.83$. In order to observe the controlling process clearly, the control acts on the system when $n=2000$. Fig. 15(a) shows time response of the amplitude $B$ of the external sine driving signal adjusted by the adjustment algorithm. The initial value of $B$ is zero. After applying the control on the system, we find that the amplitude $B$ swiftly tends to a stable value $B^{*}=0.152618$. The controlled system is in a stable state with an external driving force $u=0.152618 \sin (\omega t)$ acted on the mass $M_{1}$. The time response of phase shift $\tau$ is shown in Fig. 15(b). The time responses of variables $\dot{x}_{1+}, x_{2}$ and $\dot{x}_{2}$ are presented in Fig. 15(d)-(e), respectively. From the simulative results shown in Fig. 15(b)-(e), we can observe that $\tau$ finally trends to $\tau_{0}$. However, the other variables $\dot{x}_{1+}, x_{2}$ and $\dot{x}_{2}$ in the Poincaré section, stabilize to new values, i.e., $X_{0}^{*}=\left(\dot{x}_{1+}^{*}, x_{2}^{*}, \dot{x}_{2}^{*}, \tau_{0}^{*}\right)^{\mathrm{T}}=(-0.419595312,-0.02899704,0.02903741,1.80213090)^{\mathrm{T}}$. A phase portrait of the system, after controlling, is shown in Fig. 16(a), time series in Fig. 16(b). The system at $\omega=2.83$, after controlling, exhibits 1-2-1 motion.

From Figs. 15 and 16, the control can suppress chaos effectively. The method needs little time from control action to chaos suppressing and the time response is very fast within forty-seven impact periods. Local zooming of the time response of phase shift $\tau$ is shown in Fig. 15(b1). 

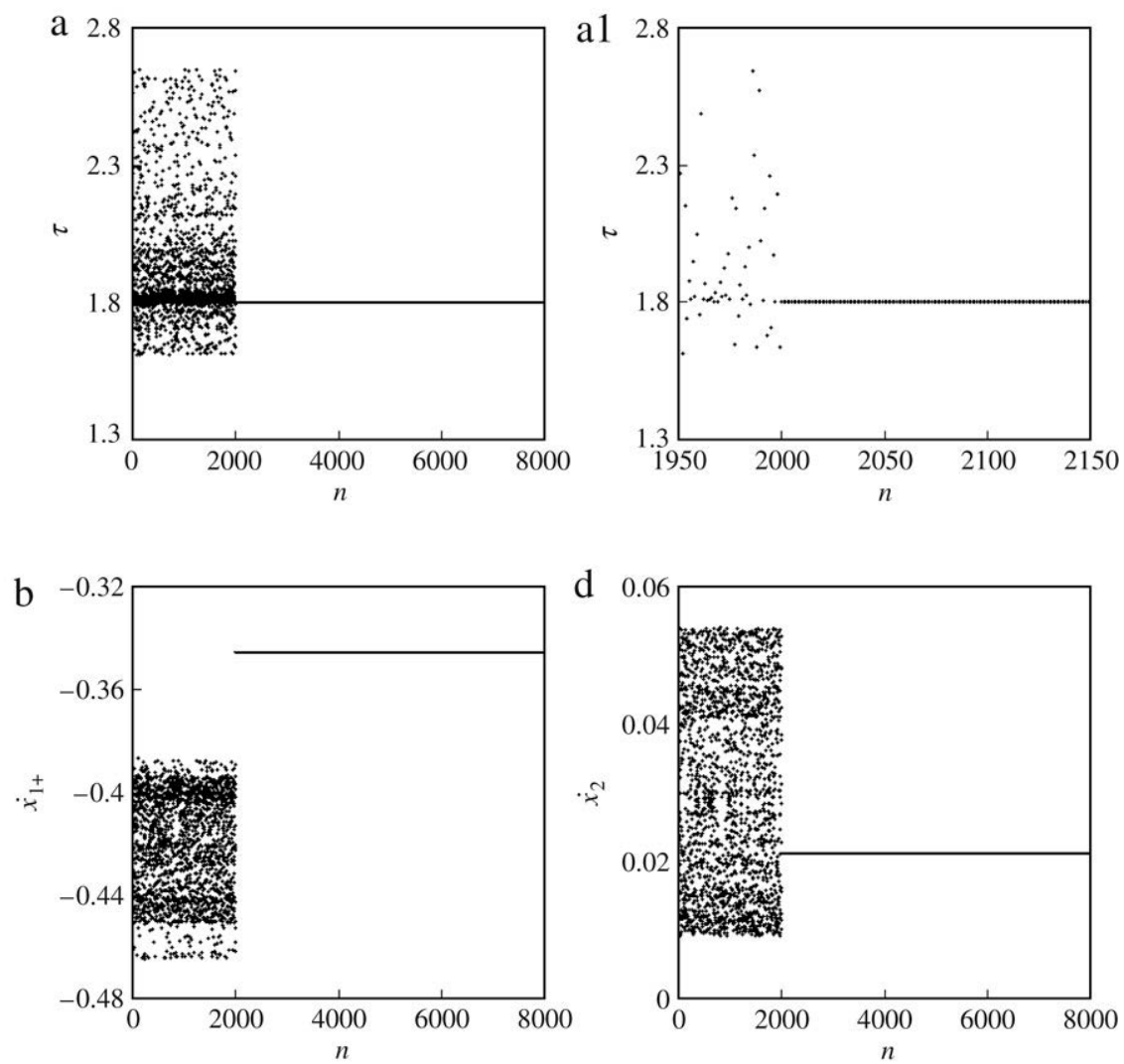

Fig. 17. Time responses of some variables (no controlling $(n<2000)$, after controlling $(n \geq 2000)), \omega=2.83$.

\subsection{Suppressing chaotic impacts by using period pulse}

The effect on the periodic impulse signal suppressing the chaotic-impact motion is observed by the presentation of an example. Consider the ideal case of $E=0$ in Fig. 3 and the stable fixed point as the control target. Once the state signals deviate from the control target, the system dynamically produces period impulse signals to suppress chaos and bifurcation.

From Fig. 6(a), when no control is applied, the system falls into chaos via Neimark-Sacker bifurcation and phase locking with decrease in the excitation frequency $\omega$.

The system parameters: $\mu_{m}=0.5, \mu_{k}=0.5, \mu_{c}=0.5, \zeta=0.1, r=0.8$ and $\delta=0.1$ are considered. The excitation frequency $\omega$ is taken as the bifurcation parameter. As we have known, the system is in stable 1-1-1 symmetric motion for $\omega \in[3.6907,5]$. Hence the projection of the motion to Poincare section $\sigma$ is a fixed point. We have given an example of a 1-1-1 symmetrical motion existing at a forcing frequency of $\omega=3.7$. A phase portrait of this periodic motion is shown in Fig. 7(a), time series in Fig. 7(b). When $\omega$ is decreased gradually, pitchfork bifurcation of 1-1-1 symmetrical motion and Neimark-Sacker bifurcation of 1-1-1 unsymmetrical motion occur in turn. Fig. 6(a) and 9 show the simulative result that the system varies from stable fixed point to Neimark-Sacker bifurcation and even chaos.

When $\omega=2.83$, the system exhibits the chaotic-impact motion, as seen in Figs. 8(d) and 9(c). Fig. 17 shows the simulative results after applying the control on the above chaotic-impact motion while $\omega=2.83$. In order to observe the controlling process clearly, the control acts on the system when $n=2000$. A phase portrait of the system, after applying the control, is shown in Fig. 18(a), time series in Fig. 18(b). The system with $\omega=2.83$, after applying the control, is found to exhibit 1-1-1 unsymmetrical motion. Comparing Fig. 8(d) with Fig. 18, owing to the control, the original chaotic-impact motion is suppressed. The method needs little time from control action to chaos suppressing and the time response is very fast within a few impact periods, as seen in local zooming of the time response of phase shift $\tau$ shown in Fig. 17(a1). 

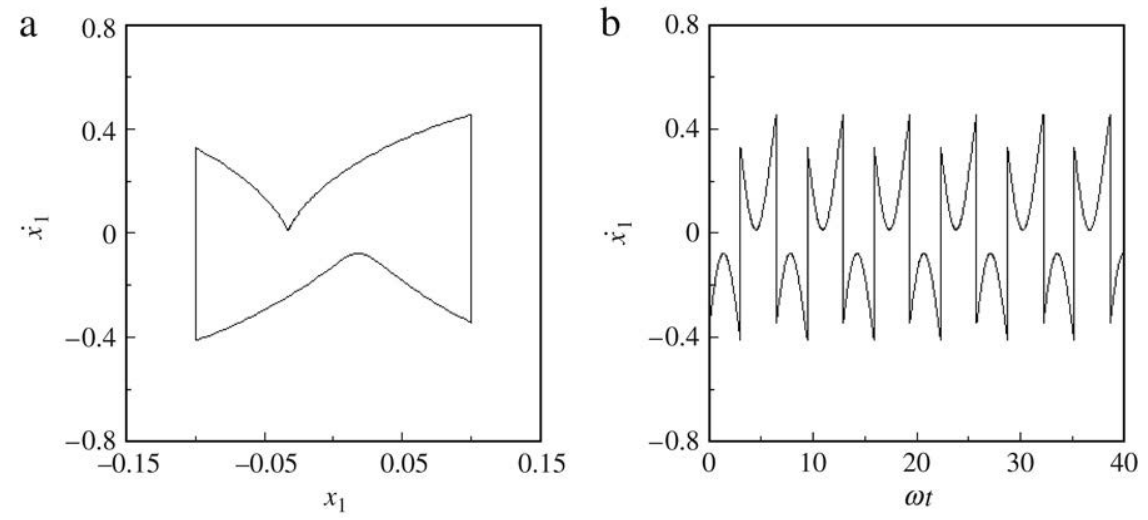

Fig. 18. Phase plane portrait and time series after controlling, $\omega=2.83$.

\subsection{Suppressing chaotic impacts based on delay feedback}

In the section we study how to suppress chaotic-impact vibration in the vibratory system with clearances when control target cannot be known beforehand. Some state variables of the previous impacts are used to suppress chaotic motion by negative feedback. The purpose of controlling is that when the chaotic-impact motion happens, it continuously feeds back some of the time-delayed state variables until the chaos is suppressed. When the chaos is suppressed, the system will be converged to the fixed point on the Poincaré section. The effect on the method is illustrated with an example of the vibratory system with a clearance.

Set the delay time as $p=1$. The non-dimensional parameters: $\mu_{m}=0.5, \mu_{k}=0.5, \mu_{c}=0.5, \zeta=0.1, r=0.8$ and $\delta=0.1$ are still chosen for observing the effect on suppressing chaos based on delay feedback. With changes of the bifurcation parameters $\omega$, the system will deviate from stable periodic orbit and convert to chaotic-impact motion. As we have known, Fig. 6(a) and (a1) respectively show bifurcation diagram of before-impact velocity and Lyapunov exponents as a function of the excitation frequency $\omega$. A chaotic attractor existing at $\omega=2.83$ is shown in Fig. 9(c).

Fig. 19 shows the suppressing of the above chaotic-impact motion, while $G=\operatorname{Diag}\left[g_{1}, g_{2}, g_{3}, g_{4}\right], g_{1}=$ $g_{2}=g_{3}=g_{4}=0.5$ and $p=1$. To illustrate the controlling effect clearly, we put the controlling at $n=2000$. The chaotic attractor in Fig. 9(c) is suppressed to the fixed point $X_{0}^{*}=\left(\dot{x}_{1+}^{*}, x_{2}^{*}, \dot{x}_{2}^{*}, \tau_{0}^{*}\right)^{\mathrm{T}}=$ $(-0.41959444,-0.02899695,0.02903516,1.80121257)^{\mathrm{T}}$ by the delay feedback control. A phase portrait of the system, after controlling, is shown in Fig. 20(a), time series in Fig. 20(b). The system at $\omega=2.83$, after controlling, is found to exhibit 1-2-1 motion. It is shown that the time delay feedback controlling method is effective in suppressing the above chaotic-impact motion.

To understand the effect of this method on suppressing such chaotic-impact motions roundly, through numerical simulations, we analyze the relationship between the feedback matrix $G=\operatorname{Diag}\left[g_{1}, g_{2}, \ldots, g_{i}, \ldots\right]$ and the result of controlling, as well as that between the time of controlling and its effect. We discuss the relationship between feedback matrix $G=\operatorname{Diag}\left[g_{1}, g_{2}, \ldots, g_{i}, \ldots\right]$ and its effect at first. While setting $G=\operatorname{Diag}\left[g_{1}, g_{2}, g_{3}, g_{4}\right]$ and $g_{1}=g_{2}=g_{3}=g_{4}=g$ in the region of $(0,1)$, we decrease $g$ from 0.98 gradually. It is seen that either large or small $g$ will prolong the converging time. Fig. 21 is the simulative results of the responses of the system's transitional process with different $g$ while other conditions are unchanged. The case of phase shift $\tau$ is only illustrated in Fig. 21, and other variables are the same. By looking at the time responses of transitional process in Figs. 19(a1) and 21, one can find that the time delay feedback controlling with $g=0.3-0.5$ is more effective in suppressing the above chaotic-impact motion.

The simulative result shows that if the $g$ is too large, the system may converge to an attracting invariant circle, rather than converge to a fixed point. For this system, if $g>0.9677$, it cannot be converged to the fixed point. Fig. 22 shows the system variable's time responses and its projection on the Poincaré section $\sigma$ when $g=0.97$ and other conditions are unchanged. To clearly show the results on the Poincare section $\sigma$, the first 2000 points at the beginning of the $n$-axis are not shown in the Poincaré section. It is obviously observed that the system converge to an attracting invariant circle. Comparing with projected Poincaré section (Fig. 22(b) and (c)) and phase portrait (Fig. 22(d)), one can find that the attracting invariant circle on impact Poincaré section represents a quasi-periodic attractor associated 

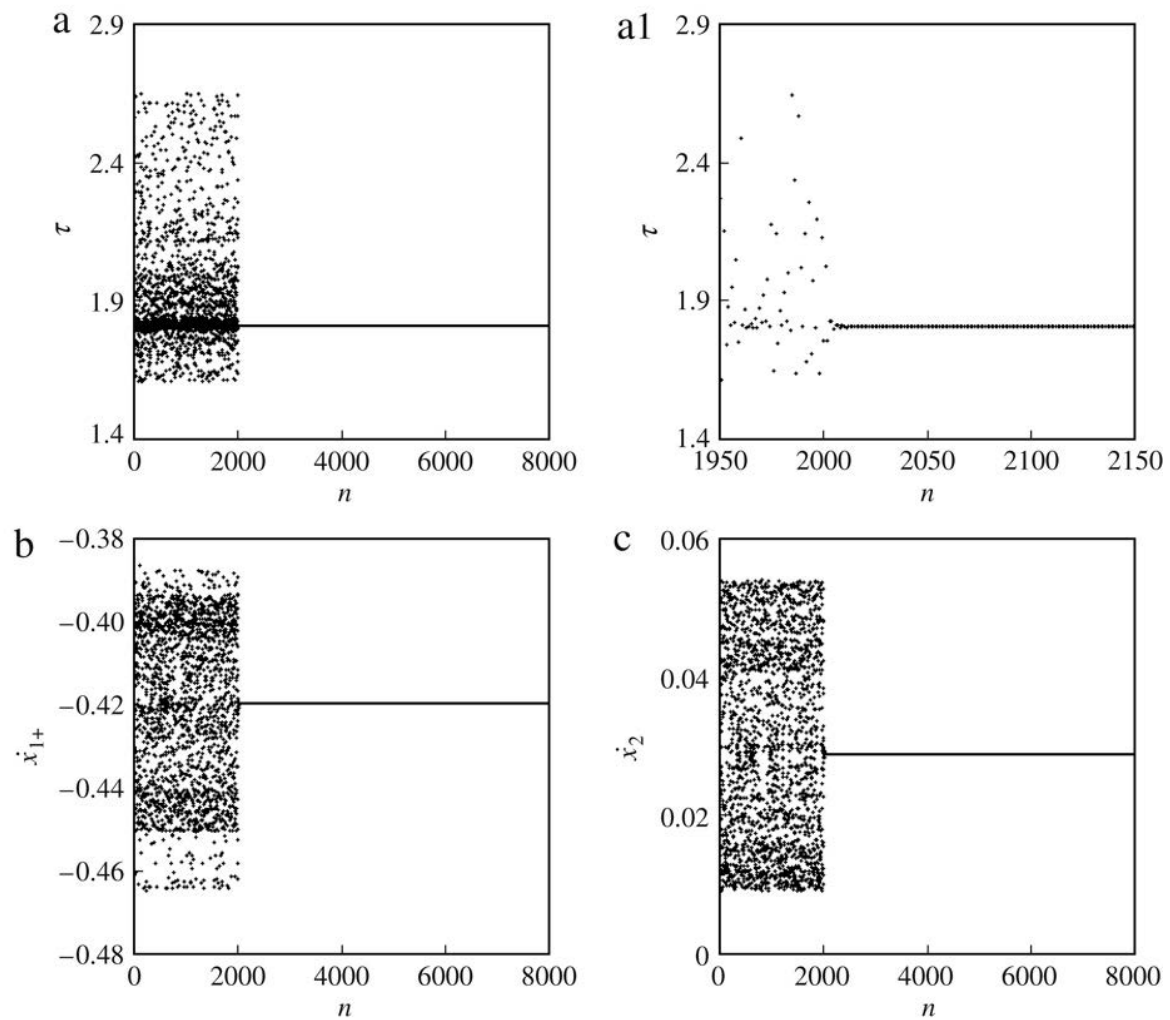

Fig. 19. Time responses of some variables (no controlling ( $n<2000)$, after controlling $(n \geq 2000)), \omega=2.83$.
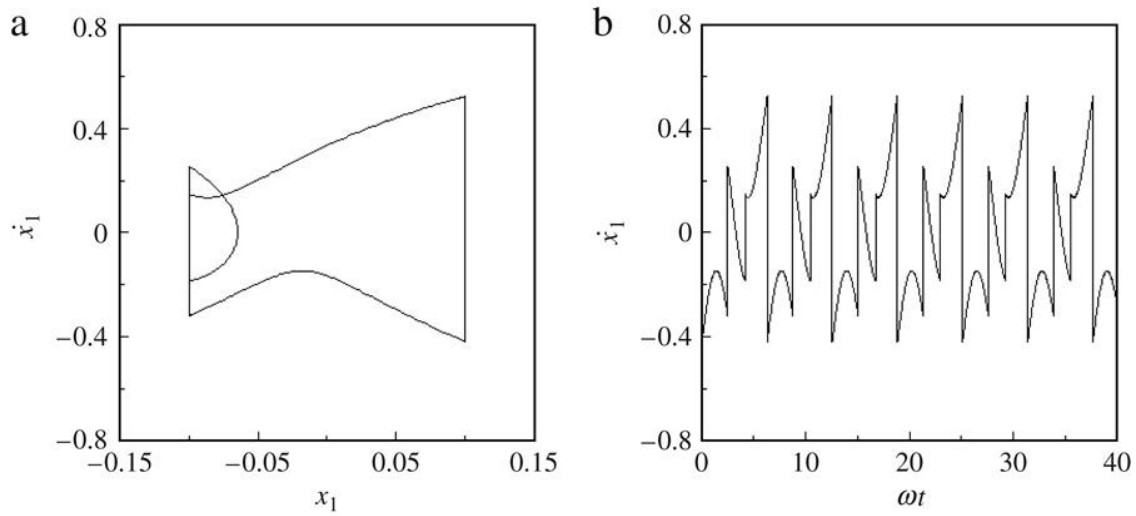

Fig. 20. Phase plane portrait and time series after controlling, $\omega=2.83$.

with the fixed point of 1-2-1 motion. The simulative result also shows that if the $g$ is too small, the system may converge to period-two fixed points. Fig. 23 shows the system variable's time responses and its phase plane portrait when $g=0.1$ and other conditions are unchanged. From Fig. 23, one can observe that the system at $\omega=2.83$, after controlling with $g=0.1$, exhibits 2-4-2 motion.

\section{Conclusions}

A two-degree-of-freedom oscillator with a clearance is considered. The maximum displacement of one of the masses is limited to a threshold value by the symmetrical stops. Impacts between the mass and the stops are 

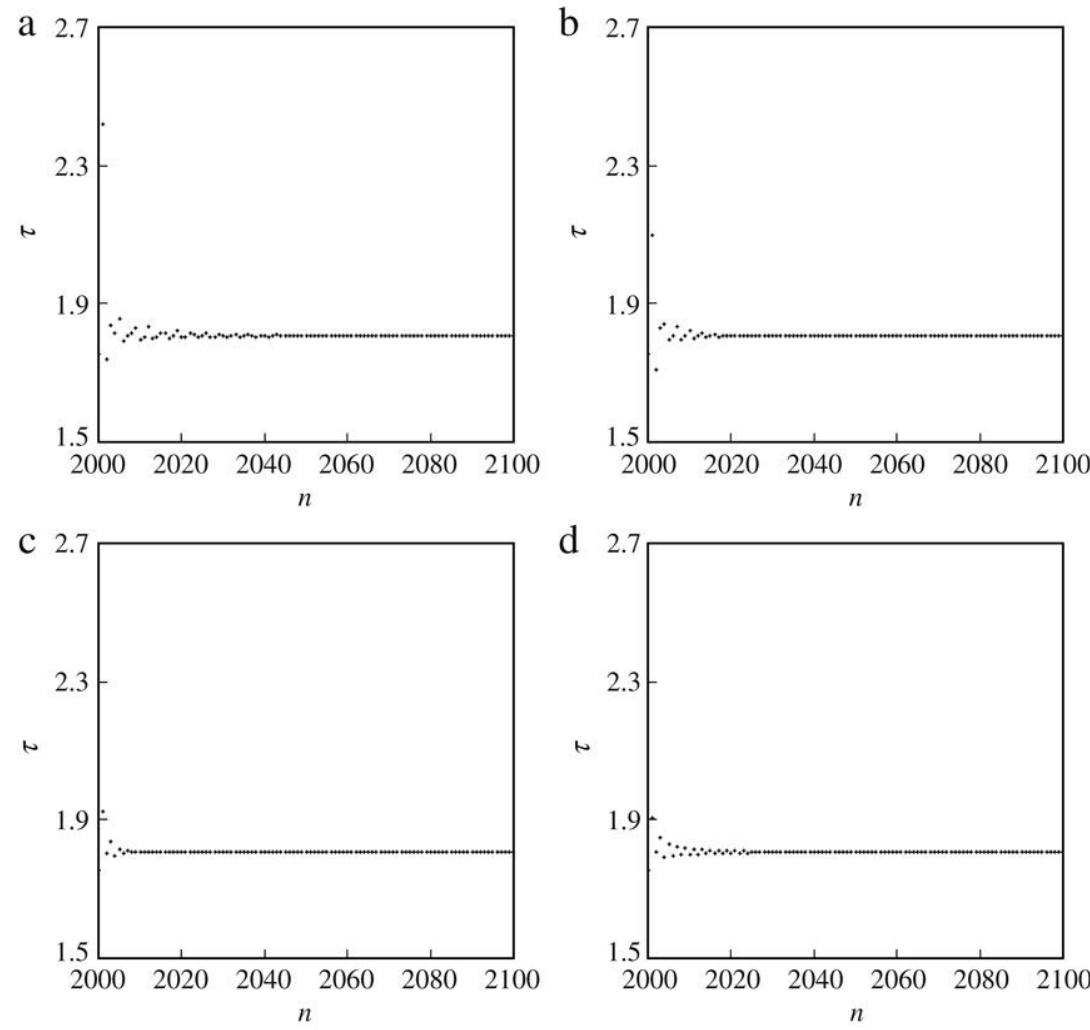

Fig. 21. The simulative results of the time responses of the system transitional process with $g$ : (a) $g=0.8$; (b) $g=0.6$; (c) $g=0.3$; (d) $g=0.25$.

described by an instantaneous coefficient of restitution. Dynamics of the system is studied with special attention to periodic-impact motions and bifurcations. Period-one double-impact symmetrical motion and transcendental impact map of the system is derived analytically. Stability and local bifurcations of the period-one double-impact symmetrical motions are analyzed by using the impact Poincaré maps. The Lyapunov exponents in vibratory systems with impacts are calculated by using the transcendental impact map. Neimark-Sacker bifurcations of period-one double-impact motions are found to occur in the system. It is well known that no Neimark-Sacker bifurcation of periodic-impact motion occurs in the single-degree-of-freedom oscillators with single stop or symmetrical stops, e.g., see Refs. [1,62]. However, not only Neimark-Sacker bifurcation associated with period-one doubleimpact symmetrical motion occurs for such two-degree-of-freedom vibratory system with a clearance, but also Neimark-Sacker bifurcation of period-one double-impact unsymmetrical motions does. The influence of the clearance $\delta$ and the excitation frequency $\omega$ on symmetrical double-impact periodic motion and bifurcations is analyzed. A series of other periodic-impact motions are found and the corresponding bifurcations are analyzed. For smaller values of clearance, period-one double-impact symmetrical motion usually undergoes pitchfork bifurcation with decrease in the forcing frequency. For large values of the clearance, period-one double-impact symmetrical motion undergoes Neimark-Sacker bifurcation with decrease in the forcing frequency. The chattering-impact vibration and the sticking phenomena are found to occur in the region of low forcing frequency, which enlarges the adverse effects such as high noise levels, wear and tear and so on. With decrease in $\omega$, non-periodic chattering-impact vibration transits to periodic one. Thereafter, sliding bifurcation of periodic chattering-impact vibration occurs. Consequently, periodic chattering-impact vibration with sticking is created. These imply that the dynamics of this system is very complex, varying from different types of periodic-impact motions to chaos, even chattering-impacting vibration and sticking, etc.

Impacting vibration arises very naturally in the vibratory systems with clearances. However, chaotic or chattering impacts are undesirable as they bring about the adverse effects such as high noise levels, wear and tear and so on. Based on the impact Poincaré map, we consider some methods of chaos suppression. The state of the vibro-impact system, just immediately after impact, is chosen as the Poincaré section, and the fixed point of 1-1-1 motion is chosen 

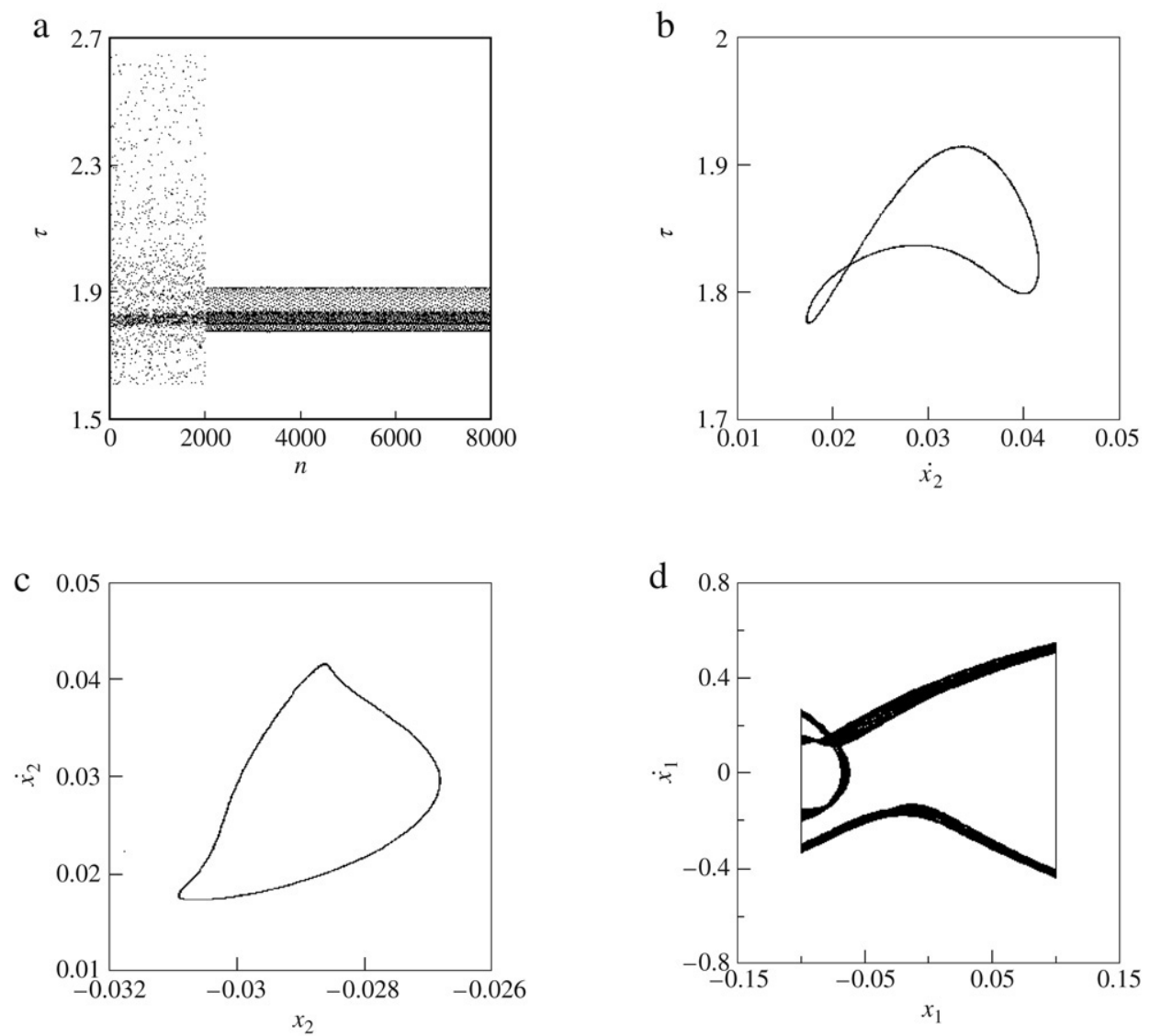

Fig. 22. The simulative result with $g=0.97$ : (a) time response of variable $\tau$ (no controlling $(n<2000)$, after controlling $(n \geq 2000)$ ); (b) and (c) projected portraits of Poincaré map (after controlling); (d) phase plane portrait (after controlling).
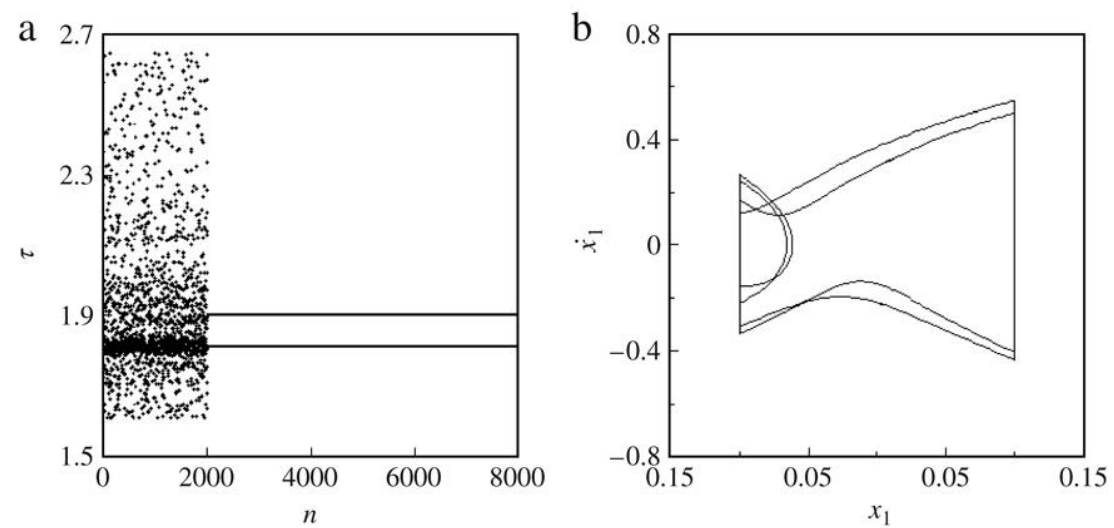

Fig. 23. The simulative result with $g=0.1$ : (a) time response of variable $\tau$ (no controlling $(n<2000)$, after controlling ( $n \geq 2000)$ ); (b) phase plane portrait (2-4-2 motion).

as the desired target. These methods are applied to the two-degree-of-freedom vibratory system with a clearance. Chaotic-impact motions of the system can be suppressed by using sine periodic force, delay feedback and feedbackbased method of period pulse to enlarge the profitable effects and minimize the adverse ones. The results show that the methods can suppress chaotic-impact motions effectively. 


\section{Acknowledgements}

The authors gratefully acknowledge the support by the National Natural Science Foundation (10572055, 50475109), the Natural Science Foundation of Gansu Province Government of China (3ZS061-A25-043 (key item)) and the 'Qing Lan' Talent Engineering by the Lanzhou Jiaotong University.

\section{Appendix}

Substituting $t=t_{n+1}$ and the formulae (8)-(11) into the general solutions (6) and (7) of Eq. (2) between the $n$ and $(n+1)$ th impacts, we can obtain the state variables $X_{n+1}=\left(\dot{x}_{1(n+1)+}, x_{2(n+1)}, \dot{x}_{2(n+1)}, \tau_{n+1}\right)^{\mathrm{T}}$ just after the $(n+1)$ th impact occurring at the stop $A$ or $\bar{A}$, which are given by Eqs. (12)-(15).

$$
\begin{aligned}
x_{1(n+1)}= & x_{1}\left(t_{n+1}\right)=\sum_{j=1}^{2} \psi_{1 j}\left(\mathrm{e}^{-\eta_{j} t_{n+1}}\left(a_{j n} \cos \omega_{d j} t_{n+1}+b_{j n} \sin \omega_{d j} t_{n+1}\right)+A_{j} \sin \left(\omega t_{n+1}+\tau_{n}\right)\right. \\
& \left.+B_{j} \cos \left(\omega t_{n+1}+\tau_{n}\right)\right),
\end{aligned}
$$

where $x_{1(n+1)}=\delta$ or $-\delta$.

By means of Eq. (A.1), we can define a function $h\left(\dot{x}_{1 n+}, x_{2 n}, \dot{x}_{2 n}, \tau_{n}, t_{n+1}\right)$ as

$$
h\left(\dot{x}_{1 n+}, x_{2 n}, \dot{x}_{2 n}, \tau_{n}, t_{n+1}\right)=x_{1}\left(t_{n+1}\right) \mp \delta=0 .
$$

Supposing $\partial h / \partial t_{n+1} \neq 0$, according to the implicit function theorem, we can solve Eq. (A.2) for

$$
t_{n+1}=t\left(\dot{x}_{1 n+}, x_{2 n}, \dot{x}_{2 n}, \tau_{n}\right) \text {. }
$$

Let $X_{n}=\left(\dot{x}_{1 n+}, x_{2 n}, \dot{x}_{2 n}, \tau_{n}\right)^{\mathrm{T}}=\left(y_{1}, y_{2}, y_{3}, y_{4}\right)^{\mathrm{T}}$. The partial derivative of $t_{n+1}$ with respect to $\dot{x}_{1 n+}, x_{2 n}, \dot{x}_{2 n}$ and $\tau_{n}$ can be computed by

$$
\frac{\partial t_{n+1}}{\partial y_{j}}=-\frac{\partial h}{\partial y_{j}} /\left(\frac{\partial h}{\partial t_{n+1}}\right), \quad j=1,2,3,4 .
$$

Inserting the formulae (8)-(11) and Eq. (A.3) into Eqs. (12)-(15), we get the transcendental impact map derived from the equations of motion, between impacts, supplemented by transition conditions at the instants of impacts, which is expressed briefly by

$$
\left\{\begin{array}{l}
\dot{x}_{1(n+1)+}=\tilde{f}_{1}\left(\dot{x}_{1 n+}, x_{2 n}, \dot{x}_{2 n}, \tau_{n}, t_{n+1}\right) \stackrel{\text { Def }}{=} f_{1}\left(\dot{x}_{1 n+}, x_{2 n}, \dot{x}_{2 n}, \tau_{n}\right), \\
x_{2(n+1)}=\tilde{f}_{2}\left(\dot{x}_{1 n+}, x_{2 n}, \dot{x}_{2 n}, \tau_{n}, t_{n+1}\right) \stackrel{\text { Def }}{=} f_{2}\left(\dot{x}_{1 n+}, x_{2 n}, \dot{x}_{2 n}, \tau_{n}\right), \\
\dot{x}_{2(n+1)}=\tilde{f}_{3}\left(\dot{x}_{1 n+}, x_{2 n}, \dot{x}_{2 n}, \tau_{n}, t_{n+1}\right) \stackrel{\text { Def }}{=} f_{3}\left(\dot{x}_{1 n+}, x_{2 n}, \dot{x}_{2 n}, \tau_{n}\right), \\
\tau_{n+1} \stackrel{\text { Def }}{=} f_{4}\left(\dot{x}_{1 n+}, x_{2 n}, \dot{x}_{2 n}, \tau_{n}\right) .
\end{array}\right.
$$

The components in the matrix (16) are obtained by

$$
\frac{\partial f_{j}}{\partial y_{i}}=\frac{\partial \tilde{f}_{j}}{\partial y_{i}}+\frac{\partial \tilde{f}_{j}}{\partial t_{n+1}} \cdot \frac{\partial t_{n+1}}{\partial y_{i}}, \quad(i, j=1,2,3,4) .
$$

Although the components in the matrix (16) are derived, the state variables $\dot{x}_{1 n+}, x_{2 n}, \dot{x}_{2 n}$ and $\tau_{n}$, just after the $n$th impact, are still included in all components in the matrix. It is important to note, that the state variables just after the $n$th impact cannot be analytically derived by combining Eqs. (6) and (7) and the matching conditions at the impact instants due to the transcendental equations. Due to the unknown state variables, we cannot directly use the Jacobian matrix to compute its eigenvalues. However, it is easy to obtain the state variables $\dot{x}_{1 n+}, x_{2 n}, \dot{x}_{2 n}$ and $\tau_{n}$ just after the $n$th impact numerically. Inserting the state variables $\dot{x}_{1 n+}, x_{2 n}, \dot{x}_{2 n}$ and $\tau_{n}$ numerically obtained into the components of the Jacobian matrix (16), one can compute the eigenvalues of the matrix. 


\section{References}

[1] S.W. Shaw, P.J. Holmes, A periodically forced piecewise linear oscillator, Journal of Sound and Vibration 90 (1) (1983) $129-155$.

[2] G.W. Luo, Y.L. Zhang, J.G. Zhang, Codimension two bifurcations of fixed points in a class of vibratory systems with rigid stops, Nonlinear Analysis: Real World Applications 8 (4) (2007) 1272-1292.

[3] J.O. Aidanpää, B.R. Gupta, Periodic and chaotic behaviour of a threshold-limited two-degree-of-freedom system, Journal of Sound and Vibration 165 (2) (1993) 305-327.

[4] D.D. Quinn, K. Bairavarasu, Near-simultaneous impacts, International Journal of Impact Engineering 32 (6) (2006) $889-904$.

[5] J. Knudsen, A.R Massih, Dynamic stability of weakly damped oscillators with elastic impacts and wear, Journal of Sound and Vibration 263 (1) (2003) 175-204.

[6] Albert C.J. Luo, Period-doubling induced chaotic motion in the LR model of a horizontal impact oscillator, Chaos, Solitons and Fractals 19 (2004) 823-839.

[7] M.F. Dimentberg, D.V. Iourtchenko, Stochastic and/or chaotic response of a vibration system to imperfectly periodic sinusoidal excitation, International Journal of Bifurcation and chaos 15 (6) (2005) 2057-2061.

[8] S.L.T de Souza, I.L. Caldas, R.L. Viana, et al., Basins of attraction changes by amplitude constraining of oscillators with limited power supply, Chaos, Solitons and Fractals 26 (4) (2005) 1211-1220.

[9] D.J. Wagg, Multiple non-smooth events in multi-degree-of-freedom vibro-impact systems, Nonlinear Dynamics 43 (1-2) (2006) 137-148.

[10] G.W. Luo, Hopf-flip bifurcations of vibratory systems with impacts, Nonlinear Analysis, Series B: Real World Applications 7 (5) (2006) $1029-1041$.

[11] G.W. Luo, Y.L. Zhang, J.G. Zhang, Codimension two bifurcations of fixed points in a class of vibratory systems with rigid stops, Nonlinear Analysis, Series B: Real World Applications 8 (4) (2007) 1272-1292.

[12] B. Blazejczyk-Okolewska, K. Czolczynski, T. Kapitaniak, Determination of geometrical conditions of assembly and impacts in classified types of mechanical systems with impacts, European Journal of Mechanics, A-Solids 24 (2) (2005) 277-291.

[13] A.B. Nordmark, Non-periodic motion caused by grazing incidence in an impact oscillator, Journal of Sound and Vibration 145 (2) (1991) 279-297.

[14] F. Peterka, J. Vacik, Transition to chaotic motion in mechanical systems with impacts, Journal of Sound and Vibration 154 (1992) 95-115.

[15] G.S. Whiston, Singularities in virbo-impact dynamics, Journal of Sound and Vibration 152 (3) (1992) 427-460.

[16] S. Foale, S.R. Bishop, Dynamical complexities of forced impacting systems, Philosophical Transactions of the Royal Society of London 338 (A) (1992) 547-556.

[17] A.P. Ivanov, Stabilization of an impact oscillator near grazing incidence owing to resonance, Journal of Sound and Vibration 162 (3) (1993) $562-565$.

[18] C. Budd, F. Dux, A. Cliffe, The effect of frequency and clearance variations on single-degree-of-freedom impact oscillators, Journal of Sound and Vibration 184 (3) (1995) 475-502.

[19] A.P. Ivanov, Bifurcation in impact systems, Chaos, Solitons and Fractal 7 (10) (1996) 1615-1634.

[20] M. Di Bernardo, M.I. Feigin, S.J. Hogan, M.E. Homer, Local analysis of C-bifurcations in N-dimensional piecewise-smooth dynamical systems, Chaos, Solitons and Fractals 10 (11) (1999) 1881-1908.

[21] K.D. Murphy, T.M. Morrison, Grazing instabilities and post-bifurcation behavior in an impacting string, Journal of the Acoustical Society of America 111 (2) (2002) 884-892.

[22] C. Halse, M. Homer, M. Di Bernardo, C-bifurcations and period-adding in one-dimensional piecewise-smooth maps, Chaos, Solitons and Fractals 18 (5) (2003) 953-976.

[23] Albert C.J. Luo, L. Chen, Periodic motions and grazing in a harmonically forced, piecewise, linear oscillator with impacts, Chaos, Solitons and Fractals 24 (2) (2005) 567-578.

[24] Albert C.J. Luo, Brandon C. Gegg, Grazing phenomena in a periodically forced, friction-induced, linear oscillator, Communications in Nonlinear Science and Numerical Simulation 11 (7) (2006) 777-802.

[25] E.E. Pavlovskaia, M. Wiercigroch, K.C. Woo, A.A. Rodger, Modeling of ground moling dynamics by an impact oscillator with a frictional slider, Meccanica 38 (2003) 85-97.

[26] E.E. Pavlovskaia, M. Wiercigroch, C. Grebogi, Two-dimensional map for impact oscillator with drift, Physical Review E 70 (2004) 036201.

[27] E.E. Pavlovskaia, M. Wiercigroch, C. Grebogi, Modeling of an impact system with a drift, Physical Review E 64 (2001) 056224.

[28] M. Wiercigroch, R.D. Neilson, M.A. Player, Material removal rate prediction for ultrasonic drilling of hard materials using an impact oscillator approach, Physics Letters A 259 (2) (1999) 91-96.

[29] S.L.T. de Souza, Ibere L. Caldas, Calculation of Lyapunov exponents in systems with impacts, Chaos, Solitons and Fractals 19 (3) (2004) 569-579.

[30] F. Peterka, T. Kotera, S. Čipera, Explanation of appearance and characteristics of intermittency chaos of the impact oscillator, Chaos, Solitons and Fractals 19 (5) (2004) 1251-1259.

[31] Albert C.J. Luo, Grazing and chaos in a periodically forced, piecewise linear system, Journal of Vibration and Acoustics 128 (2006) 28-34.

[32] D.J. Wagg, Rising phenomena and the multi-sliding bifurcation in a two-degree of freedom impact oscillator, Chaos, Solitons and Fractals 22 (2004) 541-548.

[33] D.J. Wagg, Periodic sticking motion in a two-degree-of-freedom impact oscillator, International Journal of Non-Linear Mechanics 40 (8) (2005) 1076-1087.

[34] Albert C.J. Luo, B.C. Gegg, Stick and non-stick periodic motions in periodically forced oscillators with dry friction, Journal of Sound and Vibration 291 (1-2) (2006) 132-168.

[35] H.Y. Hu, Controlling chaos of a periodically forced nonsmooth mechanical system, Acta Mechanica Sinica 11 (3) (1995) $251-258$. 
[36] H.Y. Hu, Controlling chaos of a dynamical system with discontinuous vector field, Physica D 106 (1) (1997) 1-8.

[37] J.Y. Lee, J.J. Yan, Control of impact oscillator, Chaos, Solitons and Fractals 28 (1) (2006) 136-142.

[38] S.L.T. de Souza, I.L. Caldas, Controlling chaotic orbits in mechanical systems with impacts, Chaos, Solitons and Fractals 19 (1) (2004) $171-178$.

[39] S.L.T. de Souza, M. Wiercigroch, I.L. Caldas, J.M. Balthazar, Suppressing grazing chaos in impacting system by structural nonlinearity, Chaos, Solitons and Fractals (2007) doi:10.1016/j.chaos.2007.01.022.

[40] P.B. Zinjade, A.K. Mallik, Impact damper for controlling friction-driven oscillations, Journal of Sound and Vibration 306 (1-2) (2007) 238-251.

[41] Wenli Zhao, Linze Wang, Random Fatigue and Clearance Nonlinearity of Mechanical Vibrating Systems, Science Publishing House, Beijing, China, 2006.

[42] M. Wiercigroch, V.T.W. Sin, Experimental study of base excited symmetrically piecewise linear oscillator, ASME Journal of Applied Mechanics 65 (3) (1998) 657.

[43] J.P. Meijaard, A.D. de Pater, Railway vehicle systems dynamics and chaotic vibrations, International Journal of Non-Linear Mechanics 24 (1) (1989) 1-17.

[44] G.W. Luo, J.N. Yu, H.M. Yao, J.H. Xie, Periodic-impact motions and bifurcations of the vibratory system with a clearance, Chinese Journal of Mechanical Eengineering 42 (2006) 88-94.

[45] J.H. Xie, The mathematical model for the impact hammer and global bifurcations, Acta Mechanica Sinica 29 (4) (1997) 456-463.

[46] G.W. Luo, H.M. Yao, Dynamics of a small vibro-impact pile driver, Nonlinear Analysis, Series B: Real World Applications (2007) doi:10.1016/j.nonrwa.2007.03.004.

[47] E.E. Pavlovskaia, E.V. Karpenko, M. Wiercigroch, Non-linear dynamic interactions of a Jeffcott rotor with preloaded snubber ring, Journal of Sound and Vibration 276 (2004) 361-379.

[48] E.V. Karpenko, M. Wiercigroch, E.E. Pavlovskaia, M.P. Cartmell, Piecewise approximate analytical solutions for a Jeffcott rotor with a snubber ring, International Journal of Mechanical Sciences 44 (3) (2002) 475-488.

[49] E.V. Karpenko, M. Wiercigroch, E.E. Pavlovskaia, R.D. Neilson, Experimental verification of Jeffcott rotor model with preloaded snubber ring, Journal of Sound and Vibration 298 (4-5) (2006) 907-917.

[50] D.D. Quinn, The dynamics of two parametrically excited pendula with impacts, International Journal of Bifurcation and Chaos 15 (6) (2005) 1975-1988.

[51] C.K. Sung, W.S. Yu, Dynamics of harmonically excited impact damper: Bifurcations and chaotic motion, Journal of Sound and Vibration 158 (2) (1992) 317-329.

[52] P.R.S. Han, A.C.J. Luo, Chaotic motion of a horizontal impact pair, Journal of Sound and Vibration 181 (2) (1995) $231-250$.

[53] M.S. Heiman, A.K. Bajaj, P.J. Sherman, Periodic motions and bifurcations in dynamics of an inclined impact pair, Journal of Sound and Vibration 124 (1) (1988) 55-78.

[54] C.N. Bapat, The general motion of an inclined impact damper with friction, Journal of Sound and Vibration 184 (3) (1995) $417-427$.

[55] M.R. Duncan, C.R. Wassgren, C.M. Krousgrill, The damping performance of a single particle impact damper, Journal of Sound and Vibration $286(1-2)(2005)$ 123-144.

[56] A. Kahraman, R. Singh, Non-linear dynamics of a geared rotor-bearing system with multiple clearances, Journal of Sound and Vibration 144 (3) (1991) 469-506.

[57] A. Kunert, F. Pfeiffer, Stochastic model for rattling in gear-boxes. Nonlinear dynamics in engineering system, in: W. Schiehlen (Ed.), Nonlinear Dynamics in Engineering Systems, Springer-Verlag, Berlin, Heidelberg, 1990, pp. 173-180.

[58] B. Blazejczyk-Okolewska, K. Czolczynski, T. Kapitaniak, European Journal of Mechanics A-Solids 23 (3) (2004) $517-537$.

[59] M. Di Benardo, A.R. Champneys, C.J. Budd, Grazing, skipping and sliding: analysis of the non-smooth dynamics of the dc/dc buck converter, Nonlinearity 11 (4) (1998) 858-890.

[60] M. Di Bernardo, K.H. Johansson, F. Vasca, Self-oscillations and sliding in relay feedback systems: Symmetry and bifurcations, International Journal of Bifurcation and Chaos 4 (11) (2001) 1121-1140.

[61] Albert C.J. Luo, On flow switching bifurcations in discontinuous dynamical systems, Communications in Nonlinear Science and Numerical Simulation 12 (1) (2007) 100-116.

[62] S.W. Shaw, The dynamics of a harmonically excited system having rigid amplitude constraints. Part 1, Part 2, Journal of Applied Mechanics 52 (1985) 453-464. 